\title{
REMARKS ON EXACT CONTROLLABILITY FOR THE NAVIER-STOKES EQUATIONS*
}

\author{
Oleg Yu. Imanuvilov ${ }^{1}$
}

\begin{abstract}
We study the local exact controllability problem for the Navier-Stokes equations that describe an incompressible fluid flow in a bounded domain $\Omega$ with control distributed in a subdomain $\omega \subset \Omega \subset \mathbb{R}^{n}, n \in\{2,3\}$. The result that we obtained in this paper is as follows. Suppose that $\hat{v}(t, x)$ is a given solution of the Navier-Stokes equations. Let $v_{0}(x)$ be a given initial condition and $\left\|\hat{v}(0, \cdot)-v_{0}\right\|<\varepsilon$ where $\varepsilon$ is small enough. Then there exists a locally distributed control $u$, supp $u \subset(0, T) \times \omega$ such that the solution $v(t, x)$ of the Navier-Stokes equations:
\end{abstract}

$$
\partial_{t} v-\Delta v+(v, \nabla) v=\nabla p+u+f, \operatorname{div} v=0,\left.v\right|_{\partial \Omega}=0,\left.v\right|_{t=0}=v_{0}
$$

coincides with $\hat{v}(t, x)$ at the instant $T: v(T, x) \equiv \hat{v}(T, x)$

Résumé. On étudie le problème de contrôlabilité locale exacte pour les équations de Navier-Stokes incompressibles dans un domaine $\Omega$ borné avec un contrôle réparti dans un sous-domaine $\omega \subset \Omega$ $\subset \mathbb{R}^{n}, n \in\{2,3\}$. On obtient le résultat suivant. Supposons que $\hat{v}(t, x)$ soit une solution des équations de Navier-Stokes et $v_{0}(x)$ une condition initiale telle que $\left\|\hat{v}(0, \cdot)-v_{0}\right\|<\varepsilon$ pour $\varepsilon$ assez petit. On montre alors qu'il existe un contrôle localement réparti $u, \operatorname{supp} u \subset[0, T] \times \omega$, tel que la solution $v(t, x)$ des équations de Navier-Stokes:

$$
\partial_{t} v-\Delta v+(v, \nabla) v=\nabla p+u+f, \quad \operatorname{div} v=0,\left.\quad v\right|_{\partial \Omega}=0,\left.\quad v\right|_{t=0}=v_{0}
$$

coïncide avec $\hat{v}(t, x)$ au temps $T: v(T, x) \equiv \hat{v}(T, x)$.

Mathematics Subject Classification. 35K10, 35K55, 35K60, 93B05, 93C10, 93C20.

Received August 27, 1999. Revised February 29 and September 28, 2000.

The paper is concerned on the local exact controllability of the Navier-Stokes equations, defined on the bounded domain $\Omega \subset \mathbb{R}^{n}(n=2,3)$ with boundary $\partial \Omega \in C^{2}$. More precisely, the investigated problem is as follows. Let us consider the nonstationary Navier-Stokes equations

$$
\partial_{t} v(t, x)-\Delta v(t, x)+(v, \nabla) v+\nabla p=f+\chi_{\omega} u \text { in } \Omega, \quad \operatorname{div} v=0
$$

Keywords and phrases: Locally distributed control, Navier-Stokes system.

* A part of this work was performed at the Université de Versailles. The author is grateful to Prof. J.P. Puel for hospitality and suggestions which considerably improved this paper.

1 Department of Mathematics, Iowa State University, 400 Carver Hall, Ames, IA 50011-2064, U.S.A.; e-mail: vika@iastate.edu 
with boundary and initial conditions

$$
\left.v\right|_{\Sigma}=0,\left.\quad v\right|_{t=0}=v_{0}(x)
$$

where $v(t, x)=\left(v_{1}(t, x), \ldots, v_{n}(t, x)\right)$ is a velocity of fluid, $p$ is a pressure, $f(t, x)=\left(f_{1}(t, x), \ldots, f_{n}(t, x)\right)$ is a density of external forces, $u(t, x)$ is a control distributed in some an arbitrary fixed subdomain $\omega$ of the domain $\Omega$, and $\chi_{\omega^{-}}$is a characteristic function of the set $\omega$ :

$$
\chi_{\omega}(x)= \begin{cases}1, & \text { for } x \in \omega \\ 0, & \text { for } x \in \Omega \backslash \omega .\end{cases}
$$

Let $(\hat{v}(t, x), \hat{p}(t, x))$ be a solution of the Navier-Stokes equations with the right hand side $f$ exactly same as in $(1)$ :

$$
\partial_{t} \hat{v}-\Delta \hat{v}+(\hat{v}, \nabla) \hat{v}+\nabla \hat{p}=f \text { in } \Omega, \quad \operatorname{div} \hat{v}=0,\left.\quad \hat{v}\right|_{\Sigma}=0
$$

close enough to the initial condition $v_{0}$ at the moment $t=0$

$$
\left\|v_{0}-\hat{v}(0, \cdot)\right\|_{V^{1}(\Omega)} \leq \varepsilon, \quad \text { (the parameter } \varepsilon \text { is sufficiently small) }
$$

where $V^{1}(\Omega)=\left\{v(x)=\left(v_{1}, \ldots, v_{n}\right) \in\left(W_{2}^{1}(\Omega)\right)^{n}: \operatorname{div} v=0,\left.v\right|_{\partial \Omega}=0\right\}$.

One needs to find a control $u$ such that for the given $T>0$ equality holds

$$
v(T, x)=\hat{v}(T, x) .
$$

We assume:

Condition 1. The boundary $\partial \Omega=\Gamma=\cup_{i=1}^{N} \Gamma_{i} \in C^{2},\left(\Gamma_{i} \cap \Gamma_{j}=\emptyset\right.$ for all $\left.i \neq j\right)$ where $\Gamma_{i}$ is a n-1 dimensional compact connected manifold of class $C^{2}$.

In order to formulate our results we introduce the following functional spaces

$$
\begin{gathered}
V^{0}(\Omega)=\left\{v(x)=\left(v_{1}, \ldots, v_{n}\right) \in\left(L^{2}(\Omega)\right)^{n}: \operatorname{div} v=0,\left.(v, \nu)\right|_{\partial \Omega}=0\right\}, \\
V^{1,2}(Q)=\left\{v(t, x) \in\left(W_{2}^{1,2}(Q)\right)^{n}: \operatorname{div} v=0,\left.v\right|_{\partial \Omega}=0\right\},
\end{gathered}
$$

where $\nu=\nu(x)=\left(\nu_{1}(x), \ldots, \nu_{n}(x)\right)$ is the outward unit normal to $\partial \Omega$.

The main result of this paper is the following theorem:

Theorem 1. Let $v_{0} \in V^{1}(\Omega), f \in L^{2}\left(0, T ; V^{0}(\Omega)\right)$ and suppose that the pair $(\hat{v}, \hat{p}) \in W_{\infty}^{1}\left(0, T ;\left(V^{1}(\Omega)\right.\right.$ $\left.\left.\bigcap\left(W_{\infty}^{1}(\Omega)\right)^{n}\right)\right) \times L^{2}\left(0, T ; W_{2}^{1}(\Omega)\right)$ is a given solution of the Navier-Stokes equations $(3)$ with the right hand side $f$. Then for sufficiently small $\varepsilon>0$ there exists a solution $(v, p, u) \in V^{1,2}(Q) \times L^{2}\left(0, T ; W_{2}^{1}(\Omega)\right) \times\left(L^{2}\left(Q_{\omega}\right)\right)^{n}$ to problem $(1,2,4,5)$.

The aim of this paper is to remove some technical conditions which appeared in the same result previously proved by the author in [19]. The result of the previous paper is improved now in several directions. First we omit the condition that the function $\hat{v}$ vanished in some neighborhood of the boundary $\partial \Omega$. Second, we do not assume that the domain $\Omega$ diffeomorphic to the unit sphere. And finally, now the function $\hat{v}$ is not supposed to be a steady-state solution to the Navier-Stokes equations.

This paper is organized as follows. To prove Theorem 1 we used a version of the implicit function theorem. The only one nontrivial condition to be checked is to show that the derivative of the corresponding mapping at some point is an epimorphism. In our case this problem is equivalent to the zero controllability of the linearization of the Navier-Stokes equations at the point $\hat{v}$ (see problem (3.1-3.3)). Sections 1-3 are devoted to 
this problem. One of the usual ways to solve the controllability problem is to reduce it to a observability one for the adjoint equation. So in Section 2 we introduced a linear operator (see Eq. (2.1)) which after change $t \rightarrow-t$ is formally the adjoint of the derivative of the Navier-Stokes equations at the point $\hat{v}(t, x)$. The observability problem for this operator is solved in three steps. First in Theorem 1.2 we got an appropriate estimate for the pressure $p$. Then in Theorem 2.1 we obtained a Carleman estimate for the velocity $v$ of the fluid via a weighted $L^{2}$-norm of density of external forces $f$ and $L^{2}$-norm of the pressure $p$ on the subdomain $(0, T) \times \omega$. And finally in Theorem 2.2 we proved an estimate (non Carleman type) for the velocity where in the right hand side pressure and an initial condition are absent. In the Section 3 this observability estimate was converted into the controllability result of Theorem 3.1. In Section 4 all conditions required by the implicit function theorem are checked.

We close this introductory section by mentioning some of the previous results regarding our problems. The case of the local exact controllability for the Navier-Stokes equations with boundary and local distributed control has been studied in papers $[9,11,12,21]$ and for the Boussinesq system in [10,14]. On the other hand, in pioneering works [3-5] Coron proved the global approximate controllability for the 2-D Euler equations and the 2-D NavierStokes equations with slip boundary conditions. Later this result was extended for the case of 3-dimensional Euler equation in $[15,16]$. In [6] combining results on global approximate and local exact controllability results, Coron and Fursikov obtained the global exact controllability for the Navier-Stokes system on a 2-D manifold without boundary. The similar result for the Boussinesq system on torus was obtained recently in [14]. In [7] Fabre obtained an approximate controllability of "cut off" Navier-Stokes equations.

\section{Estimate of THE PRESSURE}

It is well-known (see [30]) that for the Stokes system the pressure $p$ is a harmonic function in $x$ for each fixed $t$. If we consider the linearization of the incompressible Navier-Stokes system at the point $\hat{v}$ the pressure is the solution of the Laplace equation which right hand side some function of $\hat{v}, v$ and derivatives of these functions. Unfortunately in the case when the velocity $v$ has zero boundary conditions, there are no explicit boundary condition on the pressure $p$. To solve observability problem in this section we will prove a new Carleman estimate for the general second order elliptic equation. The main purpose of this Carleman estimate is to "minimize" (in terms of power of $s$ ) the weight which corresponds to the term with the $L^{2}$-norm of the trace of the pressure on the boundary (see estimate (1.7)). To achieve this goal we will sacrifice the weight corresponding to the $L^{2}$-norm of the right hand side of the elliptic operator. The whole chapter is devoted to establish this estimate.

Let $\omega$ be an arbitrary subdomain of $\Omega$. Denote $Q=(0, T) \times \Omega, Q_{\omega}=(0, T) \times \omega, \Sigma=(0, T) \times \partial \Omega$. In this paper we use the following functional spaces. Recall, that $W_{p}^{k}(\Omega), k \geq 0,1 \leq p<\infty$ is the Sobolev space of functions with finite norm

$$
\|u\|_{W_{p}^{k}(\Omega)}=\left(\sum_{|\alpha| \leq k} \int_{\Omega}\left|\partial^{|\alpha|} u(x) / \partial x_{1}^{\alpha_{1}} \ldots \partial x_{n}^{\alpha_{n}}\right|^{p} d x\right)^{1 / p}
$$

where $\alpha=\left(\alpha_{1}, \alpha_{2}, \ldots, \alpha_{n}\right),|\alpha|=\alpha_{1}+\cdots+\alpha_{n}$,

$$
W_{p, P e r}^{k}(K)=\left\{u \in W_{p}^{k}(K) \mid u\left(\ldots, x_{i}+2 \ell, . .\right)=u(x) i \in\{1, \ldots, n\}\right\},
$$

where $K=\prod_{i=1}^{n}[-\ell, \ell]$,

$$
\begin{gathered}
W_{2}^{1,2}(Q)=\left\{w(t, x) \mid w \in L^{2}\left(0, T ; W_{2}^{2}(\Omega)\right), \frac{\partial w}{\partial t} \in L^{2}\left(0, T ; L^{2}(\Omega)\right)\right\} \\
V^{-1}(\Omega)=\left(V^{1}(\Omega)\right)^{*}
\end{gathered}
$$


In the domain $\Omega$ we consider the elliptic equation

$$
L^{2}(Q, \rho)=\left\{v(t, x): \int_{Q} \rho v^{2} d x d t<\infty\right\}
$$

$$
\begin{gathered}
A y=-\sum_{i, j=1}^{n} \frac{\partial}{\partial x_{i}}\left(a_{i j}(x) \frac{\partial y}{\partial x_{j}}\right)+\sum_{i=1}^{n} b_{i}(x) \frac{\partial y}{\partial x_{i}}+c(x) y=f \quad \text { in } \Omega, \\
\left.y\right|_{\partial \Omega}=g .
\end{gathered}
$$

In the above problem we assume

$$
a_{i j} \in C^{2}(\bar{\Omega}), \quad a_{i j}(x)=a_{j i}(x), \quad b_{i} \in C^{1}(\bar{\Omega}), \quad c \in L^{\infty}(\Omega),
$$

where $i, j=\{1, \ldots, n\}$ and the uniform ellipticity: there exists $\beta>0$ such that

$$
a(x, \zeta, \zeta)=\sum_{i, j=1}^{n} a_{i j}(x) \zeta_{i} \zeta_{j} \geq \beta|\zeta|^{2} \quad \forall \zeta \in \mathbb{R}^{n}, \quad x \in \Omega
$$

To formulate our Carleman estimate we need a special weight function.

We have:

Lemma 1.1 $[\mathbf{2 , 2 0}]$. Let $\omega_{1} \Subset \omega$ be an arbitrary fixed subdomain of $\Omega$. Then there exists a function $\psi \in C^{2}(\bar{\Omega})$ such that

$$
\psi(x)>0 \forall x \in \Omega,\left.\psi\right|_{\partial \Omega}=0, \quad|\nabla \psi(x)|>0 \quad \forall x \in \Omega \backslash \omega_{1} .
$$

Using the function $\psi(x)$ constructed in Lemma 1.1 we introduce the function $\varphi$ by formula

$$
\varphi(x)=e^{\lambda \psi(x)},
$$

where $\lambda>1$ is a parameter to be fixed below.

Also we need the following result:

Theorem 1.1 [2, 20]. Let $(1.3,1.4)$ be fulfilled, $\psi \in C^{2}(\bar{\Omega}),|\nabla \psi(x)| \neq 0$, in $\overline{\Omega \backslash \omega_{1}}$ and let $\varphi$ be the function defined by (1.6). Then there exists a number $\hat{\lambda}>1$ such that for an arbitrary $\lambda \geq \hat{\lambda}$ there exists $s_{0}(\lambda)$ such that for each $s \geq s_{0}(\lambda)$

$$
\begin{aligned}
& \int_{\Omega}\left(s|\nabla y|^{2}+s^{3} y^{2}\right) e^{2 s \varphi} d x \leq C_{1}\left(\int_{\Omega}|A y|^{2} e^{2 s \varphi} d x+\int_{\Gamma_{1}}\left|\frac{\partial y}{\partial \nu}\right|^{2} e^{2 s \varphi} d \sigma+\int_{\omega_{1}} s^{3} y^{2} e^{2 s \varphi} d x\right) \\
& \forall y \in W_{2}^{2}(\Omega) \cap \stackrel{\circ}{W_{2}^{1}(\Omega),},
\end{aligned}
$$

where the constant $C_{1}$ depends continuously on $\lambda$ and $\Gamma_{1}=\operatorname{Int}(\partial \Omega \backslash\{x \in \partial \Omega \mid a(x, \nu, \nabla \psi) \leq 0\})$.

As it was mentioned above the main purpose of this chapter is to prove the following theorem:

Theorem 1.2. Let $(1.3,1.4)$ be fulfilled and functions $\psi, \varphi$, be defined as in $(1.5,1.6)$. Then there exists a number $\hat{\lambda}>0$ such that for an arbitrary $\lambda \geq \hat{\lambda}$ there exists $s_{0}(\lambda)$ such that for each $s \geq s_{0}(\lambda)$ the solutions 
to problem $(1.1,1.2)$ satisfy the following inequality:

$$
\int_{\Omega}\left(s^{\frac{5}{4}} y^{2}+s^{-\frac{3}{4}}|\nabla y|^{2}\right) e^{2 s \varphi} d x \leq C_{2}\left(\|g\|_{W_{2}^{\frac{1}{2}}(\partial \Omega)}^{2} e^{2 s}+\int_{\Omega} s^{-\frac{1}{4}} f^{2} e^{2 s \varphi} d x+\int_{\omega_{1}} s^{2} y^{2} e^{2 s \varphi} d x\right)
$$

where constant $C_{2}$ is independent of $s$.

To keep the proof of this theorem transparent we will separate it on several steps. To prove Theorem 1.2 let us first consider the following auxiliary problem

$$
\begin{gathered}
L z=-\sum_{i, j=1}^{n} \frac{\partial}{\partial x_{i}}\left(a_{i j}(x) \frac{\partial z}{\partial x_{j}}\right)+\sum_{i=1}^{n} b_{i}(x) \frac{\partial z}{\partial x_{i}}+c(x) z=f \quad \text { in } G, \\
z\left(x_{1}, \ldots, x_{i}+2 \ell, \ldots\right)=z\left(x_{1}, \ldots, x_{i} \ldots\right) \quad i \in\{2, \ldots, n\} \\
z\left(0, x^{\prime}\right)=g\left(x^{\prime}\right), \quad \frac{\partial z\left(-1, x^{\prime}\right)}{\partial x_{1}}=z\left(-1, x^{\prime}\right)=0,
\end{gathered}
$$

where $x^{\prime}=\left(x_{2}, \ldots, x_{n}\right), K=\prod_{i=1}^{n-1}[-\ell, \ell], G=[-1,0] \times K$, and $\ell>0$ be an arbitrary fixed number.

Assume the following condition:

Condition 1.1. We assume that

$$
\begin{aligned}
a_{11}(x) \equiv 1, \quad a_{i j}\left(x_{1}, \ldots, x_{k}+2 \ell, \ldots\right) & =a_{i j}(x), \quad b_{i}\left(x_{1}, \ldots, x_{k}+2 \ell, \ldots\right)=b_{i}(x) \\
c\left(x_{1}, \ldots, x_{k}+2 \ell, \ldots\right) & =c(x) \quad \forall i, j \in\{1, \ldots, n\}, \quad k \in\{2, \ldots, n\} \\
a_{i j} \in C^{2}(\bar{G}), \quad a_{i j}(x) & =a_{j i}(x), \quad b_{i} \in C^{1}(\bar{G}), \quad c \in L^{\infty}(G)
\end{aligned}
$$

where $i, j \in\{1, \ldots, n\}$ and the uniform ellipticity: there exists $\beta>0$ such that

$$
a(x, \zeta, \zeta)=\sum_{i, j=1}^{n} a_{i j}(x) \zeta_{i} \zeta_{j} \geq \beta|\zeta|^{2} \quad \forall \zeta \in \mathbb{R}^{n}, \quad x \in G
$$

Denote

$$
\phi\left(x_{1}\right)=e^{\lambda \tilde{\psi}\left(x_{1}\right)}, \quad \tilde{\psi}\left(x_{1}\right)=-x_{1}
$$

where $\lambda>1$ is some parameter.

Lemma 1.2. Let Condition 1.1 be fulfilled and the function $\phi$ be defined as in (1.11). Then there exists a number $\hat{\lambda}>1$ such that for an arbitrary $\lambda \geq \hat{\lambda}$ there exists $s_{0}(\lambda)$ that for $s \geq s_{0}(\lambda)$ the solutions to problem (1.8-1.10) satisfy the following inequality:

$$
\int_{G}\left(s^{\frac{1}{4}}|\nabla z|^{2}+s^{\frac{9}{4}} z^{2}\right) e^{2 s \phi} d x \leq C_{3}\left(\int_{K}\left(\left|\nabla_{x^{\prime}} g\right|^{2}+g^{2}\right) e^{2 s \phi(0)} d x^{\prime}+\int_{G} s^{-\frac{1}{4}} f^{2} e^{2 s \phi} d x\right)
$$

where constant $C_{3}$ is independent of $s$.

Proof. Denote $w=e^{s \phi} z, \tilde{f}=e^{s \phi} f$. Then by (1.8)

$$
\mathbf{L} w=e^{s \phi} L e^{-s \phi} w=\tilde{f} .
$$


The operator $\mathbf{L}$ can be written explicitly as follows

$$
\begin{aligned}
\mathbf{L} w= & -\sum_{i, j=1}^{n} \frac{\partial}{\partial x_{i}}\left(a_{i j} \frac{\partial w}{\partial x_{j}}\right)-2 s \lambda \phi a\left(x, \vec{e}_{1}, \nabla w\right)+s \lambda^{2} \phi a\left(x, \vec{e}_{1}, \vec{e}_{1}\right) w \\
& -s^{2} \lambda^{2} \phi^{2} a\left(x, \vec{e}_{1}, \vec{e}_{1}\right) w+\sum_{i=1}^{n} b_{i} \frac{\partial w}{\partial x_{i}}+c w-s \lambda \phi \sum_{i=1}^{n} \frac{\partial a_{i 1}}{\partial x_{i}} w+s \lambda \phi b_{1} w .
\end{aligned}
$$

We recall that the quadratic form $a(x, \xi, \eta)$ was defined in (1.4). We introduce the operators $L_{1}, L_{2}$ by formulas

$$
\begin{gathered}
L_{1} w=-\sum_{i, j=1}^{n} \frac{\partial}{\partial x_{i}}\left(a_{i j} \frac{\partial w}{\partial x_{j}}\right)-s^{2} \lambda^{2} \phi^{2} a\left(x, \vec{e}_{1}, \vec{e}_{1}\right) w \\
L_{2} w=-2 s \lambda \phi a\left(x, \vec{e}_{1}, \nabla w\right)+2 s \lambda^{2} \phi a\left(x, \vec{e}_{1}, \vec{e}_{1}\right) w .
\end{gathered}
$$

By (1.13-1.15), using the new notations we have

$$
L_{1} w+L_{2} w=f_{s} \quad \text { in } \quad G
$$

where

$$
f_{s}(x)=f e^{s \phi}+s \lambda^{2} \phi a\left(x, \vec{e}_{1}, \vec{e}_{1}\right) w-\sum_{i=1}^{n} b_{i} \frac{\partial w}{\partial x_{i}}-c w+s \lambda \phi \sum_{i=1}^{n} \frac{\partial a_{i 1}}{\partial x_{i}} w-s \lambda \phi b_{1} w
$$

We set $G_{\tau}=[-1, \tau] \times K, \tau \in(-1,0)$.

Taking the $L^{2}\left(G_{\tau}\right)-$ norm of both sides of (1.16) we obtain

$$
\left\|f_{s}\right\|_{L^{2}\left(G_{\tau}\right)}^{2}=\left\|L_{1} w\right\|_{L^{2}\left(G_{\tau}\right)}^{2}+\left\|L_{2} w\right\|_{L^{2}\left(G_{\tau}\right)}^{2}+2\left(L_{1} w, L_{2} w\right)_{L^{2}\left(G_{\tau}\right)}
$$

By (1.14) and (1.15) we have the following equality:

$$
\begin{aligned}
\left(L_{1} w, L_{2} w\right)_{L^{2}\left(G_{\tau}\right)}= & \left(-\sum_{i, j=1}^{n} \frac{\partial}{\partial x_{i}}\left(a_{i j} \frac{\partial w}{\partial x_{j}}\right)-\lambda^{2} s^{2} \phi^{2} a\left(x, \vec{e}_{1}, \vec{e}_{1}\right) w, 2 s \lambda^{2} \phi a\left(x, \vec{e}_{1}, \vec{e}_{1}\right) w\right)_{L^{2}\left(G_{\tau}\right)} \\
& +\int_{G_{\tau}} 2 \lambda^{3} s^{3} \phi^{3} a\left(x, \vec{e}_{1}, \vec{e}_{1}\right) w a\left(x, \vec{e}_{1}, \nabla w\right) d x \\
& +\int_{G_{\tau}} \sum_{i, j=1}^{n} \frac{\partial}{\partial x_{i}}\left(a_{i j} \frac{\partial w}{\partial x_{j}}\right) 2 s \lambda \phi a\left(x, \vec{e}_{1}, \nabla w\right) d x=A_{0}+A_{1}+A_{2} .
\end{aligned}
$$


Integrating by parts in the first term of the right hand side of (1.19) we obtain

$$
\begin{aligned}
A_{0}= & \left(-\sum_{i, j=1}^{n} \frac{\partial}{\partial x_{i}}\left(a_{i j} \frac{\partial w}{\partial x_{j}}\right)-\lambda^{2} s^{2} \phi^{2} a\left(x, \vec{e}_{1}, \vec{e}_{1}\right) w, 2 s \lambda^{2} \phi a\left(x, \vec{e}_{1}, \vec{e}_{1}\right) w\right)_{L^{2}\left(G_{\tau}\right)} \\
= & \int_{G_{\tau}}\left(-2 s^{3} \phi^{3} \lambda^{4} a\left(x, \vec{e}_{1}, \vec{e}_{1}\right)^{2} w^{2}+2 s \lambda^{2} \phi a\left(x, \vec{e}_{1}, \vec{e}_{1}\right) a(x, \nabla w, \nabla w)\right. \\
& \left.+2 s \lambda^{2} w \sum_{i, j=1}^{n} a_{i j} \frac{\partial w}{\partial x_{j}} \frac{\partial}{\partial x_{i}}\left(\phi a\left(x, \vec{e}_{1}, \vec{e}_{1}\right)\right)\right) d x \\
& -\int_{\{\tau\} \times K} 2 s \lambda^{2} \phi a\left(x, \vec{e}_{1}, \vec{e}_{1}\right) w a\left(x, \vec{e}_{1}, \nabla w\right) d x^{\prime} .
\end{aligned}
$$

Integrating by parts in the second term of the right-hand-side of (1.19), we have

$$
\begin{aligned}
A_{1}= & \int_{G_{\tau}} 2 \lambda^{3} s^{3} \phi^{3} w a\left(x, \vec{e}_{1}, \vec{e}_{1}\right) a\left(x, \vec{e}_{1}, \nabla w\right) d x \\
= & \int_{G_{\tau}} \lambda^{3} s^{3} \phi^{3} a\left(x, \vec{e}_{1}, \vec{e}_{1}\right) a\left(x, \vec{e}_{1}, \nabla w^{2}\right) d x \\
= & \int_{G_{\tau}}\left(3 \lambda^{4} s^{3} \phi^{3} a\left(x, \vec{e}_{1}, \vec{e}_{1}\right)^{2} w^{2}-w^{2} \phi^{3} \lambda^{3} s^{3} \sum_{i=1}^{n} \frac{\partial}{\partial x_{i}}\left(a_{i 1} a\left(x, \vec{e}_{1}, \vec{e}_{1}\right)\right)\right) d x \\
& +\int_{\{\tau\} \times K} \lambda^{3} s^{3} \phi^{3} a\left(x, \vec{e}_{1}, \vec{e}_{1}\right)^{2} w^{2} d x^{\prime} .
\end{aligned}
$$

Finally, integrating by parts for the third term of right-hand-side of (1.19) we have

$$
\begin{aligned}
A_{2}= & \int_{G_{\tau}} \sum_{i, j=1}^{n} \frac{\partial}{\partial x_{i}}\left(a_{i j} \frac{\partial w}{\partial x_{j}}\right) 2 s \lambda \phi a\left(x, \vec{e}_{1}, \nabla w\right) d x \\
= & \int_{G_{\tau}}\left(2 s \lambda^{2} \phi a\left(x, \vec{e}_{1}, \nabla w\right)^{2}-2 s \lambda \phi \sum_{i, j=1}^{n}\left(a_{i j} \frac{\partial w}{\partial x_{i}} \sum_{\ell=1}^{n} \frac{\partial a_{1 \ell}}{\partial x_{j}} \frac{\partial w}{\partial x_{\ell}}\right)\right. \\
& \left.-2 s \lambda \phi \sum_{i, j=1}^{n} a_{i j} \frac{\partial w}{\partial x_{i}} \sum_{\ell=1}^{n} a_{1 \ell} \frac{\partial^{2} w}{\partial x_{j} \partial x_{\ell}}\right) d x+\int_{\{\tau\} \times K} 2 s \lambda \phi a\left(x, \vec{e}_{1}, \nabla w\right)^{2} d x^{\prime} \\
= & \int_{G_{\tau}}\left(2 s \lambda^{2} \phi a\left(x, \vec{e}_{1}, \nabla w\right)^{2}-2 s \lambda \phi \sum_{i, j=1}^{n}\left(a_{i j} \frac{\partial w}{\partial x_{i}} \sum_{\ell=1}^{n} \frac{\partial a_{1 \ell}}{\partial x_{j}} \frac{\partial w}{\partial x_{\ell}}\right)\right. \\
& \left.+s \lambda \phi \sum_{\ell=1}^{n} a_{1 \ell} \sum_{i, j=1}^{n} \frac{\partial a_{i j}}{\partial x_{\ell}} \frac{\partial w}{\partial x_{i}} \frac{\partial w}{\partial x_{j}}-s \lambda \phi \sum_{\ell=1}^{n} a_{1 \ell} \frac{\partial}{\partial x_{\ell}} \sum_{i, j=1}^{n} a_{i j} \frac{\partial w}{\partial x_{i}} \frac{\partial w}{\partial x_{j}}\right) d x \\
& +\int_{\{\tau\} \times K} 2 s \lambda \phi a\left(x, \vec{e}_{1}, \nabla w\right)^{2} d x^{\prime} .
\end{aligned}
$$


Integrating in (1.22) by parts once again, we obtain

$$
\begin{aligned}
A_{2}= & \int_{G}\left(2 s \lambda^{2} \phi a\left(x, \vec{e}_{1}, \nabla w\right)^{2}-2 s \lambda \phi \sum_{i, j=1}^{n}\left(a_{i j} \frac{\partial w}{\partial x_{i}} \sum_{\ell=1}^{n} \frac{\partial a_{1 \ell}}{\partial x_{j}} \frac{\partial w}{\partial x_{\ell}}\right)+s \lambda \phi \sum_{\ell=1}^{n} a_{1 \ell} \sum_{i, j=1}^{n} \frac{\partial a_{i j}}{\partial x_{\ell}} \frac{\partial w}{\partial x_{i}} \frac{\partial w}{\partial x_{j}}\right. \\
& \left.-s \lambda^{2} \phi a\left(x, \vec{e}_{1}, \vec{e}_{1}\right) a(x, \nabla w, \nabla w)+a(x, \nabla w, \nabla w) s \lambda \phi \sum_{\ell=1}^{n} \frac{\partial a_{1 \ell}}{\partial x_{\ell}}\right) d x \\
& +\int_{\{\tau\} \times K}\left(-s \lambda \phi a\left(x, \vec{e}_{1}, \vec{e}_{1}\right) a(x, \nabla w, \nabla w)+2 s \lambda \phi a\left(x, \vec{e}_{1}, \nabla w\right)^{2}\right) d x^{\prime} .
\end{aligned}
$$

Using (1.19-1.21) and (1.23) one can rewrite (1.18) as follows

$$
\begin{aligned}
\left\|f_{s}\right\|_{L^{2}\left(G_{\tau}\right)}^{2}= & \left\|L_{1} w\right\|_{L^{2}\left(G_{\tau}\right)}^{2}+\left\|L_{2} w\right\|_{L^{2}\left(G_{\tau}\right)}^{2} \\
& +2 \int_{G_{\tau}}\left(\lambda^{4} s^{3} \phi^{3} a\left(x, \vec{e}_{1}, \vec{e}_{1}\right)^{2} w^{2}+s \lambda^{2} \phi a\left(x, \vec{e}_{1}, \vec{e}_{1}\right) a(x, \nabla w, \nabla w)\right. \\
& \left.+2 s \lambda^{2} \phi a\left(x, \vec{e}_{1}, \nabla w\right)^{2}\right) d x+\mathcal{S}(\tau, w)+X_{1}(\tau)+X_{2}(\tau),
\end{aligned}
$$

where

$$
\begin{aligned}
\mathcal{S}(\tau, w)= & 2 \int_{\{\tau\} \times K}\left(2 s \lambda \phi a\left(x, \vec{e}_{1}, \nabla w\right)^{2}-s \lambda \phi a\left(x, \vec{e}_{1}, \vec{e}_{1}\right) a(x, \nabla w, \nabla w)\right. \\
& \left.-2 s \lambda^{2} \phi a\left(x, \vec{e}_{1}, \vec{e}_{1}\right) w a\left(x, \vec{e}_{1}, \nabla w\right)+s^{3} \lambda^{3} \phi^{3} a\left(x, \vec{e}_{1}, \vec{e}_{1}\right)^{2} w^{2}\right) d x^{\prime}
\end{aligned}
$$

and

$$
\begin{gathered}
X_{1}(\tau)=2 \int_{G_{\tau}}\left(-2 s \lambda \phi \sum_{i, j=1}^{n}\left(a_{i j} \frac{\partial w}{\partial x_{i}} \sum_{\ell=1}^{n} \frac{\partial a_{1 \ell}}{\partial x_{j}} \frac{\partial w}{\partial x_{\ell}}\right)-w^{2} \phi^{3} \lambda^{3} s^{3} \sum_{i=1}^{n} \frac{\partial}{\partial x_{i}}\left(a_{i 1} a\left(x, \vec{e}_{1}, \vec{e}_{1}\right)\right)\right. \\
\left.+s \lambda \phi \sum_{\ell=1}^{n} a_{1 \ell} \sum_{i, j=1}^{n} \frac{\partial a_{i j}}{\partial x_{\ell}} \frac{\partial w}{\partial x_{i}} \frac{\partial w}{\partial x_{j}}+a(x, \nabla w, \nabla w) s \lambda \phi \sum_{\ell=1}^{n} \frac{\partial a_{1 \ell}}{\partial x_{\ell}}\right) d x \\
X_{2}=2 \int_{G_{\tau}} 2 s \lambda^{2} w \sum_{i, j=1}^{n} a_{i j} \frac{\partial w}{\partial x_{j}} \frac{\partial}{\partial x_{i}}\left(\phi a\left(x, \vec{e}_{1}, \vec{e}_{1}\right)\right) d x .
\end{gathered}
$$

Taking the parameter $\hat{\lambda}$ sufficiently large we have

$$
\begin{aligned}
\left\|f_{s}\right\|_{L^{2}\left(G_{\tau}\right)}^{2} \geq & \left\|L_{2} w\right\|_{L^{2}\left(G_{\tau}\right)}^{2}+\left\|L_{1} w\right\|_{L^{2}\left(G_{\tau}\right)}^{2} \\
& +\frac{11}{8} \int_{G_{\tau}}\left(\lambda^{4} s^{3} \phi^{3} a\left(x, \vec{e}_{1}, \vec{e}_{1}\right)^{2} w^{2}+s \lambda^{2} \phi a\left(x, \vec{e}_{1}, \vec{e}_{1}\right) a(x, \nabla w, \nabla w)\right. \\
& \left.+2 s \lambda^{2} \phi a\left(x, \vec{e}_{1}, \nabla w\right)^{2}\right) d x+\mathcal{S}(\tau, w)+X_{2}(\tau)
\end{aligned}
$$

for all $\lambda>\hat{\lambda}$ and $s>1$.

Now let $\lambda>\hat{\lambda}$ be fixed. Taking the parameter $s_{0}(\lambda)$ sufficiently large we obtain from the previous inequality

$$
\begin{aligned}
\left\|f_{s}\right\|_{L^{2}\left(G_{\tau}\right)}^{2} \geq & \left\|L_{2} w\right\|_{L^{2}\left(G_{\tau}\right)}^{2}+\left\|L_{1} w\right\|_{L^{2}\left(G_{\tau}\right)}^{2} \\
& +\int_{G_{\tau}}\left(\lambda^{4} s^{3} \phi^{3} a\left(x, \vec{e}_{1}, \vec{e}_{1}\right)^{2} w^{2}+s \lambda^{2} \phi a\left(x, \vec{e}_{1}, \vec{e}_{1}\right) a(x, \nabla w, \nabla w)\right. \\
& \left.+2 s \lambda^{2} \phi a\left(x, \vec{e}_{1}, \nabla w\right)^{2}\right) d x+\mathcal{S}(\tau, w) \quad \forall s \geq s_{0}(\lambda) .
\end{aligned}
$$


By $(1.15,1.10)$ and Cauchy-Bunyakovskii inequality

$$
\frac{1}{4}\left\|L_{2} w\right\|_{L^{2}\left(G_{\tau}\right)}^{2}+\frac{1}{4} \int_{G_{\tau}} s^{3} \lambda^{4} \phi^{3} a\left(x, \vec{e}_{1}, \vec{e}_{1}\right)^{2} w^{2} d x \geq \kappa s^{\frac{5}{2}} \int_{K} w\left(\tau, x^{\prime}\right)^{2} d x^{\prime} \quad \forall \tau \in[-1,0],
$$

where constant $\kappa>0$ is independent of $s, \tau$.

Denote by

$$
\tau^{*}(w)=\max _{\tau \in[-1,0]}\left\{\tau \mid \mathcal{S}(\tau, w) \geq-\kappa s^{\frac{5}{2}} \int_{K} w\left(\tau, x^{\prime}\right)^{2} d x^{\prime}\right\}
$$

By $(1.25-1.27)$

$$
\begin{aligned}
\left\|f_{s}\right\|_{L^{2}\left(G_{\tau^{*}}\right)}^{2} \geq & \frac{3}{4} \int_{G_{\tau^{*}}}\left(\lambda^{4} s^{3} \phi^{3} a\left(x, \vec{e}_{1}, \vec{e}_{1}\right)^{2} w^{2}+s \lambda^{2} \phi a\left(x, \vec{e}_{1}, \vec{e}_{1}\right) a(x, \nabla w, \nabla w)\right. \\
& \left.+2 s \lambda^{2} \phi a\left(x, \vec{e}_{1}, \nabla w\right)^{2}\right) d x \quad \forall s \geq s_{0}(\lambda) .
\end{aligned}
$$

If $\tau^{*}(w)=0$ the lemma is proved. Now let us consider the case $\tau^{*}(w)<0$. Multiplying $(1.16)$ by $s a_{11} w$ scalary in $L^{2}(G)$ and integrating by parts we have

$$
\begin{aligned}
\int_{G} \tilde{f} s a_{11} w d x= & \int_{G}\left(s a\left(x, \vec{e}_{1}, \vec{e}_{1}\right) a(x, \nabla w, \nabla w)-s^{3} \lambda^{2} \phi^{2} a\left(x, \vec{e}_{1}, \vec{e}_{1}\right)^{2} w^{2}\right) d x \\
& +\int_{G} s a\left(x, \nabla w, \nabla a_{11}\right) w d x-\int_{\{0\} \times K} s a_{11} a\left(x, \vec{e}_{1}, \nabla w\right) w d x^{\prime} \\
& -\int_{\{0\} \times K} s^{2} \lambda \phi a_{11} a\left(x, \vec{e}_{1}, \vec{e}_{1}\right) w^{2} d x^{\prime}+\frac{1}{2} \int_{\{0\} \times K} s a_{11} b_{1} w^{2} d x^{\prime}+X_{3},
\end{aligned}
$$

where

$$
\begin{aligned}
X_{3}= & \int_{G}\left(s^{2} \lambda^{2} \phi a\left(x, \vec{e}_{1}, \vec{e}_{1}\right)^{2} w^{2}+\sum_{j=1}^{n} s^{2} \lambda \frac{\partial}{\partial x_{j}}\left(\phi a_{11} a_{1 j}\right) w^{2}\right. \\
& \left.+s a_{11}\left(-\sum_{i=1}^{n} \frac{\partial a_{i 1}}{\partial x_{i}} s \lambda \phi+c+s \lambda \phi b_{1}\right) w^{2}-\frac{s}{2} \sum_{i=1}^{n} \frac{\partial\left(b_{i} a_{11}\right)}{\partial x_{i}} w^{2}\right) d x .
\end{aligned}
$$

Obviously

$$
\left|X_{3}\right| \leq C_{4} s^{2} \int_{G} w^{2} d x
$$

Note that

$$
\begin{aligned}
& -\int_{\{0\} \times K} s a_{11} a\left(x, \vec{e}_{1}, \nabla w\right) w d x^{\prime}-\int_{\{0\} \times K} s^{2} \lambda \phi a_{11} a\left(x, \vec{e}_{1}, \vec{e}_{1}\right) w^{2} d x^{\prime} \\
= & -\int_{\{0\} \times K} s a_{11} a\left(x, \vec{e}_{1}, \nabla z e^{s \phi}-s \lambda \phi \vec{e}_{1} w\right) w d x^{\prime} \\
& -\int_{\{0\} \times K} s^{2} \lambda \phi a_{11} a\left(x, \vec{e}_{1}, \vec{e}_{1}\right) w^{2} d x^{\prime}=-\int_{\{0\} \times K} s a_{11} a\left(x, \vec{e}_{1}, \nabla z\right) w e^{s \phi} d x^{\prime} \\
= & -\int_{\{0\} \times K} s a_{11}^{2} \frac{\partial z}{\partial x_{1}} e^{s \phi} w d x^{\prime}+s \int_{\{0\} \times K} \sum_{j=2}^{n} \frac{1}{2} \frac{\partial\left(a_{11} a_{1 j}\right)}{\partial x_{j}} w^{2} d x^{\prime} .
\end{aligned}
$$


Denote

$$
X_{4}=\frac{1}{2} \int_{\{0\} \times K} s a_{11} b_{1} w^{2} d x^{\prime}+s \int_{\{0\} \times K} \sum_{j=2}^{n} \frac{1}{2} \frac{\partial\left(a_{1 j} a_{11}\right)}{\partial x_{j}} w^{2} d x^{\prime}
$$

Using (1.31) one could rewrite (1.29) as

$$
\begin{aligned}
\int_{G} s a_{11} \tilde{f} w d x= & \int_{G}\left(s a\left(x, \vec{e}_{1}, \vec{e}_{1}\right) a(x, \nabla w, \nabla w)-s^{3} \lambda^{2} \phi^{2} a\left(x, \vec{e}_{1}, \vec{e}_{1}\right)^{2} w^{2}\right) d x \\
& +\int_{G} s a\left(x, \nabla w, \nabla a_{11}\right) w d x-\int_{\{0\} \times K} s a_{11}^{2} \frac{\partial z}{\partial x_{1}} w e^{s \phi} d x^{\prime}+X_{3}+X_{4} .
\end{aligned}
$$

One could rewrite (1.33) as

$$
\begin{aligned}
& -\int_{-1}^{0} \frac{1}{2 \lambda \phi} S\left(x_{1}, w\right) d x_{1}+\int_{G}\left(2 s a\left(x, \vec{e}_{1}, \nabla w\right)^{2}-2 s \lambda a\left(x, \vec{e}_{1}, \vec{e}_{1}\right) w a(x, \vec{e}, \nabla w)\right) d x \\
= & \int_{G} s a_{11} \tilde{f} w d x+\int_{\{0\} \times K} s a_{11}^{2} \frac{\partial z}{\partial x_{1}} w e^{s \phi} d x^{\prime}-X_{3}-X_{4}-\int_{G} s a\left(x, \nabla w, \nabla a_{11}\right) w d x .
\end{aligned}
$$

From this equality, by (1.27) we have

$$
\begin{aligned}
\hat{\kappa}_{1} \int_{\left(\tau^{*}, 0\right) \times K} s^{\frac{5}{2}} w^{2} d x & -\int_{-1}^{\tau^{*}} \frac{1}{2 \lambda \phi} S\left(x_{1}, w\right) d x_{1}+\int_{G} 2 s a\left(x, \vec{e}_{1}, \nabla w\right)^{2} d x \\
\leq & \int_{G} 2 s \lambda a\left(x, \vec{e}_{1}, \vec{e}_{1}\right) w a(x, \vec{e}, \nabla w) d x+\int_{G} s a_{11} \tilde{f} w d x \\
& +\int_{\{0\} \times K} s a_{11}^{2} \frac{\partial z}{\partial x_{1}} w e^{s \phi} d x^{\prime}-\int_{G} s a\left(x, \nabla w, \nabla a_{11}\right) w d x-X_{3}-X_{4},
\end{aligned}
$$

where $\hat{\kappa}_{1}=\kappa /\left(2 \lambda e^{\lambda}\right)$. Hence by (1.28) we have

$$
\begin{aligned}
\hat{\kappa}_{2} \int_{G}\left(s^{\frac{5}{2}} w^{2}+s a\left(x, \nabla w, \vec{e}_{1}\right)^{2}\right) d x \leq & C_{5}\left(\int_{\{0\} \times K} s\left|a_{11}^{2} \frac{\partial z}{\partial x_{1}} w\right| e^{s \phi} d x^{\prime}\right. \\
& \left.+\left|X_{3}\right|+\left|X_{4}\right|+\int_{G}\left(|\nabla w|^{2}+s^{2} w^{2}\right) d x+s\left|\int_{G} a_{11} \tilde{f} w d x\right|+\|\tilde{f}\|_{L^{2}(G)}^{2}\right) \\
\leq & \varepsilon \int_{\{0\} \times K} s^{\frac{7}{4}} w^{2} d x^{\prime}+C_{6}\left(\frac{1}{\varepsilon} \int_{\{0\} \times K} s^{\frac{1}{4}}\left|\frac{\partial z}{\partial x_{1}}\right|^{2} e^{2 s \phi} d x^{\prime}+\|\tilde{f}\|_{L^{2}(G)}^{2}\right. \\
& \left.+\int_{G}\left(|\nabla w|^{2}+s^{2} w^{2}\right) d x+\left|X_{3}\right|+\left|X_{4}\right|\right) \quad \forall s \geq s_{0}(\lambda),
\end{aligned}
$$

where $\hat{\kappa}_{2}=\frac{1}{2} \min \left\{\hat{\kappa}_{1}, 2\right\}$ is some constant independent of $s$. Note that by $(1.10)$

$$
\int_{G}\left(s^{\frac{5}{2}} w^{2}+s a\left(x, \vec{e}_{1}, \nabla w\right)^{2}\right) d x \geq \hat{\kappa}_{3} s^{\frac{7}{4}} \int_{K} w^{2}\left(0, x^{\prime}\right) d x^{\prime},
$$

where $\hat{\kappa}_{3}>0$ is independent of $w, s$. 
Taking in (1.34) parameter $\varepsilon$ sufficiently small and increasing $s_{0}(\lambda)$ if necessary, by $(1.30,1.35)$ we obtain

$$
\begin{gathered}
\int_{G}\left(s^{\frac{5}{2}} w^{2}+s a\left(x, \vec{e}_{1}, \nabla w\right)^{2}\right) d x \leq C_{7}\left(\left|X_{4}\right|+\|\tilde{f}\|_{L^{2}(G)}^{2}+\int_{G}|\nabla w|^{2} d x\right. \\
\left.+s^{\frac{1}{4}} \int_{\{0\} \times K}\left|\frac{\partial z}{\partial x_{1}}\right|^{2} e^{2 s \phi} d x^{\prime}\right) \quad \forall s \geq s_{0}(\lambda) .
\end{gathered}
$$

Increasing parameter $s_{0}(\lambda)$ if it is necessary we obtain from $(1.35,1.36)$

$$
\int_{G}\left(s^{\frac{5}{2}} w^{2}+s a\left(x, \vec{e}_{1}, \nabla w\right)^{2}\right) d x \leq C_{8}\left(\|\tilde{f}\|_{L^{2}(G)}^{2}+\int_{G}|\nabla w|^{2} d x+s^{\frac{1}{4}} \int_{\{0\} \times K}\left|\frac{\partial z}{\partial x_{1}}\right|^{2} e^{2 s \phi} d x^{\prime}\right) \quad \forall s \geq s_{0}(\lambda) .
$$

Since

$$
\mathcal{S}(0, w) \leq 0,
$$

for all values of the parameter $s$ large enough we have

$$
\int_{K}\left|\frac{\partial z\left(0, x^{\prime}\right)}{\partial x_{1}}\right|^{2} e^{2 s \phi(0)} d x^{\prime} \leq C_{9} \int_{K}\left(\left|\nabla_{x^{\prime}} g\right|^{2}+g^{2}\right) e^{2 s \phi(0)} d x^{\prime}
$$

By $(1.37,1.38)$

$$
\int_{G}\left(s^{\frac{5}{2}} w^{2}+s a\left(x, \vec{e}_{1}, \nabla w\right)^{2}\right) d x \leq C_{10}\left(\|\tilde{f}\|_{L^{2}(G)}^{2}+\int_{G}|\nabla w|^{2} d x+s^{\frac{1}{4}} \int_{K}\left(\left|\nabla g_{x^{\prime}}\right|^{2}+g^{2}\right) e^{2 s \phi(0)} d x^{\prime}\right) \quad \forall s \geq s_{0}(\lambda) .
$$

By (1.33)

$$
\begin{aligned}
\int_{G} s^{\frac{1}{2}}|\nabla w|^{2} d x \leq & C_{11}\left(\int_{G} s^{\frac{5}{2}} w^{2} d x+\int_{\{0\} \times K} s^{\frac{1}{2}}\left|\frac{\partial z}{\partial x_{1}} e^{s \phi} w\right| d x^{\prime}\right. \\
& \left.+\|\tilde{f}\|_{L^{2}(G)}^{2}+\int_{G}|\nabla w|^{2} d x+\left|X_{3}\right|+\left|X_{4}\right|\right) \quad \forall s \geq s_{0}(\lambda) .
\end{aligned}
$$

From this inequality, by $(1.30,1.32,1.38,1.39)$ we obtain $(1.12)$.

In the right hand side of estimate (1.12) we have the $W_{2}^{1}$-norm of the function $g$. This norm is too strong for our purposes. So now we would like to obtain from this estimate the similar one but with the $L^{2}$-norm in the right hand side First we formulate the following result:

Proposition 1.1. Let $g=0$, Condition 1.1 be fulfilled and function $\phi$ be defined as in (1.11). Then there exists a number $\hat{\lambda}>0$ such that for an arbitrary $\lambda \geq \hat{\lambda}$ there exists $s_{0}(\lambda)$ such that for each $s \geq s_{0}(\lambda)$ the solutions of problem (1.8-1.10) satisfy the following inequality:

$$
\int_{G}\left(s^{1-2 \ell}|\nabla z|^{2}+s^{3-2 \ell}|z|^{2}\right) e^{2 s \phi} d x \leq C_{12} \int_{-1}^{0}\left\|f\left(x_{1}, \cdot\right)\right\|_{W_{2}^{-\ell}(K)}^{2} e^{2 s \phi} d x_{1}, \quad \forall \ell \in[0,1]
$$

where constant $C_{12}$ is independent of $s$.

The proof of this proposition is exactly the same as the proof of Theorem 2.1 as we can see from [22]. 
Now we are ready to prove the following lemma:

Lemma 1.3. Let Condition 1.1 be fulfilled and function $\phi$ be defined as in (1.11). Then there exists a number $\hat{\lambda}>0$ such that for an arbitrary $\lambda \geq \hat{\lambda}$ there exists $s_{0}(\lambda)$ such that for each $s \geq s_{0}(\lambda)$ the solutions of problem (1.8-1.10) satisfy the following inequality:

$$
\begin{aligned}
\int_{G} s^{\frac{1}{4}}|z|^{2} e^{2 s \phi} d x & +s^{\frac{1}{4}} \int_{-1}^{0}\left(\left\|\frac{\partial z}{\partial x_{1}}\left(x_{1}, \cdot\right)\right\|_{W_{2}^{-1}(K)}^{2}+s^{\frac{9}{4}}\left\|Z\left(x_{1}, \cdot\right)\right\|_{W_{2}^{-1}(K)}^{2}\right) e^{2 s \phi\left(x_{1}\right)} d x_{1} \\
& \leq C_{12}\left(\int_{K} g^{2} e^{2 s \phi(0)} d x^{\prime}+\int_{-1}^{0} s^{-\frac{1}{4}}\left\|f\left(x_{1}, \cdot\right)\right\|_{W_{2}^{-1}(K)}^{2} e^{2 s \phi} d x_{1}\right)
\end{aligned}
$$

where constant $C_{12}$ is independent of $s$.

Proof. Denote $P=-\sum_{i=2}^{n} \frac{\partial^{2}}{\partial x_{i}^{2}}+1$. There exists $P^{-1}: W_{2, P e r}^{s}(K) \rightarrow W_{2, P e r}^{s+2}(K)$ for all $s \in\{0,-1\}$. Let $R_{1}=P^{-1}, R_{i}=\frac{\partial}{\partial x_{i}} P^{-1} i \in\{2, \ldots, n\}$ and $u_{i}=R_{i} z, f_{i}=R_{i} f$. Obviously

$$
\sum_{i=2}^{n}-\frac{\partial u_{i}}{\partial x_{i}}+u_{1}=z \quad \text { in } K
$$

and

$$
\begin{gathered}
\left\|z\left(x_{1}, \cdot\right)\right\|_{L^{2}(K)} \leq C_{13} \sum_{i=1}^{n}\left\|u_{i}\left(x_{1}, \cdot\right)\right\|_{W_{2}^{1}(K)} ; \\
\left\|\frac{\partial z\left(x_{1}, \cdot\right)}{\partial x_{1}}\right\|_{W_{2}^{-1}(K)} \leq C_{13} \sum_{i=1}^{n}\left\|\frac{\partial u_{i}\left(x_{1}, \cdot\right)}{\partial x_{1}}\right\|_{L^{2}(K)} ; \\
\left\|z\left(x_{1}, \cdot\right)\right\|_{W_{2}^{-1}(K)} \leq C_{14} \sum_{i=1}^{n}\left\|u_{i}\left(x_{1}, \cdot\right)\right\|_{L^{2}(K)} .
\end{gathered}
$$

By $(1.8-1.10)$

$$
\begin{gathered}
L u_{i}+\left[L, R_{i}\right] z=f_{i} \text { in } G, \\
u_{i}\left(0, x^{\prime}\right)=R_{i} g, \quad u_{i}\left(x_{1}, . ., x_{j}+2 \ell, . .\right)=u_{i}\left(x_{1}, \ldots, x_{j}, . .\right) \quad j \in\{2, \ldots, n\}, \\
u_{i}\left(-1, x^{\prime}\right)=\frac{\partial u_{i}\left(-1, x^{\prime}\right)}{\partial x_{1}}=0 \quad \text { in } K,
\end{gathered}
$$

where $[A, B]=B A-A B$ is the commutator of operators $A$ and $B, x^{\prime}=\left(x_{2}, \ldots, x_{n}\right)$. Note that

$$
\left\|e^{s \phi}\left[L, R_{i}\right] z\left(x_{1}, \cdot\right)\right\|_{L^{2}(K)} \leq C_{15}\left(\left\|z\left(x_{1}, \cdot\right) e^{s \phi}\right\|_{L^{2}(K)}+\left\|\frac{\partial z\left(x_{1}, \cdot\right)}{\partial x_{1}} e^{s \phi}\right\|_{W_{2}^{-1}(K)}\right)
$$


where the constant $C_{15}$ is independent of $s$ and $x_{1}$. Then by (1.46) and Lemma 1.2 there exists $\hat{\lambda}>1$ such that for all $\lambda>\hat{\lambda}$ there exists $s_{0}(\lambda)$ such that

$$
\begin{aligned}
\int_{G}\left(s^{\frac{1}{4}}\left|\nabla u_{i}\right|^{2}\right. & \left.+s^{\frac{9}{4}} u_{i}^{2}\right) e^{2 s \phi} d x \leq C_{16}\left(\int_{K}|g|^{2} e^{2 s \phi(0)} d x^{\prime}\right. \\
& +\int_{-1}^{0} s^{-\frac{1}{4}}\left\|\frac{\partial z\left(x_{1}, \cdot\right)}{\partial x_{1}}\right\|_{W_{2}^{-1}(K)}^{2} e^{2 s \phi\left(x_{1}\right)} d x_{1}+\int_{G} s^{-\frac{1}{4}}|z|^{2} e^{2 s \phi} d x \\
& \left.+\int_{-1}^{0} s^{-\frac{1}{4}} e^{2 s \phi\left(x_{1}\right)}\left\|f\left(x_{1}, \cdot\right)\right\|_{W_{2}^{-1}(K)}^{2} d x_{1}\right) \quad \forall s \geq s_{0}(\lambda) .
\end{aligned}
$$

By $(1.42,1.47)$ for all $s \geq s_{0}$

$$
\begin{aligned}
\int_{G} s^{\frac{1}{4}} z^{2} e^{2 s \phi} d x & +\int_{-1}^{0}\left(s^{\frac{1}{4}}\left\|\frac{\partial z\left(x_{1}, \cdot\right)}{\partial x_{1}}\right\|_{W_{2}^{-1}(K)}^{2}+s^{\frac{9}{4}}\left\|z\left(x_{1}, \cdot\right)\right\|_{W_{2}^{-1}(K)}^{2}\right) e^{2 s \phi\left(x_{1}\right)} d x_{1} \\
\leq & C_{17} \sum_{i=1}^{n} \int_{G}\left(s^{\frac{1}{4}}\left|\nabla u_{i}\right|^{2}+s^{\frac{9}{4}} u_{i}^{2}\right) e^{2 s \phi} d x \\
\leq & C_{18}\left(\int_{K} g^{2} e^{2 s \phi(0)} d x^{\prime}+\int_{G} s^{-\frac{1}{4}} z^{2} e^{2 s \phi} d x\right. \\
& +\int_{-1}^{0} s^{-\frac{1}{4}}\left\|\frac{\partial z\left(x_{1}, \cdot\right)}{\partial x_{1}}\right\|_{W_{2}^{-1}(K)}^{2} e^{2 s \phi\left(x_{1}\right)} d x_{1} \\
& \left.+\int_{-1}^{0} s^{-\frac{1}{4}} e^{2 s \phi\left(x_{1}\right)}\left\|f\left(x_{1}, \cdot\right)\right\|_{W_{2}^{-1}(K)}^{2} d x_{1}\right) .
\end{aligned}
$$

Then by increasing the parameter $s_{0}(\lambda)$ if necessary we obtain

$$
\begin{aligned}
\int_{G} s^{\frac{1}{4}} z^{2} e^{2 s \phi} d x & +\int_{-1}^{0}\left(s^{\frac{1}{4}}\left\|\frac{\partial z\left(x_{1}, \cdot\right)}{\partial x_{1}}\right\|_{W_{2}^{-1}(K)}^{2}+s^{\frac{9}{4}}\left\|z\left(x_{1}, \cdot\right)\right\|_{W_{2}^{-1}(K)}^{2}\right) e^{2 s \phi\left(x_{1}\right)} d x_{1} \\
\leq & C_{18} \sum_{i=1} \int_{G}\left(s^{\frac{1}{4}}\left|\nabla u_{i}\right|^{2}+s^{\frac{9}{4}} u_{i}^{2}\right) e^{2 s \phi} d x \leq C_{19}\left(\int_{K} g^{2} e^{2 s \phi(0)} d x^{\prime}\right. \\
& \left.+\int_{-1}^{0} s^{-\frac{1}{4}} e^{2 s \phi\left(x_{1}\right)}\left\|f\left(x_{1}, \cdot\right)\right\|_{W_{2}^{-1}(K)}^{2} d x_{1}\right)
\end{aligned}
$$

for all $s \geq s_{0}$. The proof of this lemma is complete.

We have:

Lemma 1.4. Let Condition 1.1 be fulfilled and function $\phi$ be defined as in (1.11). Then there exists a number $\hat{\lambda}>0$ such that for an arbitrary $\lambda \geq \hat{\lambda}$ there exists $s_{0}(\lambda)$ such that for each $s \geq s_{0}(\lambda)$ the solutions of problem (1.8-1.10) satisfy the following inequality:

$$
\begin{aligned}
\int_{G}\left(\left(s^{\frac{3}{4}} x_{1}^{20}\right.\right. & \left.\left.+s^{-\frac{3}{4}}\right)|\nabla z|^{2}+s^{\frac{5}{4}}|z|^{2}\right) e^{2 s \phi} d x+s^{\frac{1}{4}} \int_{-1}^{0}\left\|\frac{\partial z}{\partial x_{1}}\left(x_{1}, \cdot\right)\right\|_{W_{2}^{-\frac{1}{2}}(K)}^{2} e^{2 s \phi\left(x_{1}\right)} d x_{1} \\
& \leq C_{25}\left(\|g\|_{W_{2}^{\frac{1}{2}}(K)}^{2} e^{2 s}+\int_{G} s^{-\frac{1}{4}} x_{1}^{10}|f|^{2} e^{2 s \phi} d x+\int_{-1}^{0} s^{-\frac{1}{4}}\left\|f\left(x_{1}, \cdot\right)\right\|_{W_{2}^{-\frac{1}{2}}(K)}^{2} e^{2 s \phi} d x_{1}\right),
\end{aligned}
$$

where constant $C_{25}$ is independent of $s$. 
Proof. Let $\tilde{z}=\Lambda z=\int_{\mathbb{R}^{n-1}} \frac{\mu\left(x^{\prime}\right) e^{i<x^{\prime}, \xi^{\prime}>}}{\left(1+\left|\xi^{\prime}\right|^{2}\right)^{\frac{1}{4}}} \widehat{\mu_{1} z} d \xi^{\prime}$, where $\xi^{\prime}=\left(\xi_{2}, \ldots, \xi_{n}\right), \mu\left(x^{\prime}\right) \in C_{0}^{\infty}\left(K_{1}\right), K_{1}=\Pi_{i=2}^{n}[-2 \ell, 2 \ell]$, $\mu_{1}\left(x^{\prime}\right) \in C_{0}^{\infty}\left(K_{2}\right), K_{2}=\Pi_{i=2}^{n}\left[-\frac{3}{2} \ell, \frac{3}{2} \ell\right], \mu_{1},\left.\mu\right|_{K}=1$. Since $z$ is the periodic function in variable $x^{\prime}$ we have

$$
\begin{gathered}
\|z\|_{W_{2}^{\frac{1}{2}}(K)} \leq C_{26}\left(\|\tilde{z}\|_{W_{2}^{1}\left(K_{1}\right)}+\|z\|_{W_{2}^{-1}(K)}\right), \\
\left\|\frac{\partial z}{\partial x_{1}}\right\|_{W_{2}^{-\frac{1}{2}(K)}} \leq C_{27}\left(\left\|\frac{\partial \tilde{z}}{\partial x_{1}}\right\|_{W_{2}^{1}\left(K_{1}\right)}+\left\|\frac{\partial z}{\partial x_{1}}\right\|_{W_{2}^{-1}(K)}\right) .
\end{gathered}
$$

By (1.40) we obtain from these estimates

$$
\begin{aligned}
\left\|z e^{s \phi}\right\|_{L^{2}\left(-1,0 ; W_{2}^{\frac{1}{2}}(K)\right)}^{2} \leq & C_{28}\left(\left\|\tilde{z} e^{s \phi}\right\|_{L^{2}\left(-1,0 ; W_{2}^{1}\left(K_{1}\right)\right)}^{2}\right. \\
& +s^{-\frac{1}{4}}\left(\int_{K} g^{2} e^{2 s \phi(0)} d x^{\prime}+\int_{-1}^{0} s^{-\frac{1}{4}}\left\|f\left(x_{1}, \cdot\right)\right\|_{W_{2}^{-1}(K)}^{2} e^{2 s \phi} d x_{1}\right) . \\
\left\|\frac{\partial z}{\partial x_{1}} e^{s \phi}\right\|_{L^{2}\left(-1,0 ; W_{2}^{-\frac{1}{2}}(K)\right)}^{2} \leq & C_{29}\left(\left\|\frac{\partial \tilde{z}}{\partial x_{1}} e^{s \phi}\right\|_{L^{2}\left(-1,0 ; W_{2}^{1}\left(K_{1}\right)\right)}^{2}\right. \\
& \left.+s^{-\frac{1}{4}}\left(\int_{K} g^{2} e^{2 s \phi(0)} d x^{\prime}+\int_{-1}^{0} s^{-\frac{1}{4}}\left\|f\left(x_{1}, \cdot\right)\right\|_{W_{2}^{-1}(K)}^{2} e^{2 s \phi} d x_{1}\right)\right) .
\end{aligned}
$$

By $(1.8-1.10)$

$$
\begin{gathered}
L \tilde{z}+[L, \Lambda] z=\Lambda f \quad \text { in } G_{1}=[-1,0] \times K_{1}, \\
\tilde{z}\left(0, x^{\prime}\right)=\Lambda g, \quad \tilde{z}\left(x_{1}, . ., x_{j}+4 \ell, . .\right)=\tilde{z}\left(x_{1}, \ldots, x_{j}, . .\right) \quad j \in\{2, \ldots, n\} \\
\tilde{z}\left(-1, x^{\prime}\right)=\frac{\partial \tilde{z}\left(-1, x^{\prime}\right)}{\partial x_{1}}=0 \quad \text { in } K_{1} .
\end{gathered}
$$

Note that (see [30])

$$
\|[L, \Lambda] z\|_{L^{2}\left(K_{1}\right)} \leq C_{30}\left(\|z\|_{W_{2}^{\frac{1}{2}(K)}}+\left\|\frac{\partial z}{\partial x_{1}}\right\|_{W_{2}^{-\frac{1}{2}}(K)}\right) .
$$

Applying to problem (1.52-1.54) the Carleman estimate (1.12) and keeping in mind (1.55) we have

$$
\begin{aligned}
\int_{G_{1}}\left(s^{\frac{1}{4}}|\nabla \tilde{z}|^{2}+s^{\frac{9}{4}} \tilde{z}^{2}\right) e^{2 s \phi} d x \leq & C_{31}\left(\int_{K_{1}}\left(\left|\nabla_{x^{\prime}} \Lambda g\right|^{2}+|\Lambda g|^{2}\right) e^{2 s \phi(0)} d x^{\prime}\right. \\
& \left.+\int_{G_{1}}\left(s^{-\frac{1}{4}}|\Lambda f|^{2}+|[L, \Lambda] z|^{2}\right) e^{2 s \phi} d x\right) \\
\leq & C_{32}\left(\|g\|_{W_{2}^{\frac{1}{2}}(K)}^{2} e^{2 s}+\int_{G_{1}} s^{-\frac{1}{4}}|\Lambda f|^{2} e^{2 s \phi} d x\right. \\
& \left.+s^{-\frac{1}{4}} \int_{-1}^{0}\left(\|z\|_{W_{2}^{\frac{1}{2}}(K)}^{2}+\left\|\frac{\partial z}{\partial x_{1}}\right\|_{W_{2}^{-\frac{1}{2}}(K)}^{2}\right) e^{2 s \phi} d x_{1}\right) .
\end{aligned}
$$


From this inequality, thanks to $(1.40,1.50,1.51)$ we obtain

$$
\begin{aligned}
\int_{G} s^{\frac{5}{4}}|z|^{2} e^{2 s \phi} d x & +s^{\frac{1}{4}} \int_{-1}^{0}\left\|\frac{\partial z}{\partial x_{1}}\left(x_{1}, \cdot\right)\right\|_{W_{2}^{-\frac{1}{2}}(K)}^{2} e^{2 s \phi\left(x_{1}\right)} d x_{1} \\
\leq & C_{33}\left(\|g\|_{W_{2}^{\frac{1}{2}}(K)}^{2} e^{2 s}+\int_{-1}^{0} s^{-\frac{1}{4}}\left\|f\left(x_{1}, \cdot\right)\right\|_{W_{2}}^{2}(K)\right.
\end{aligned}
$$

where constant $C_{33}$ is independent of $s$.

Multiplying (1.8) by $s^{-\frac{3}{4}} z e^{2 s \phi}$ scalary in $L^{2}(G)$ we have

$$
\begin{aligned}
\int_{G} s^{-\frac{3}{4}} a(x, \nabla z, \nabla z) e^{2 s \phi} d x= & -2 \int_{G} s^{\frac{1}{4}} \lambda \phi z a(x, \nabla z, \nabla \psi) e^{2 s \phi} d x-\int_{K} s^{-\frac{3}{4}} a(x, \nu, \nabla z) z e^{2 s \phi(0)} d x^{\prime} \\
& +\int_{G} s^{-\frac{3}{4}}\left(f-c z-\sum_{i=1}^{n} b_{i} \frac{\partial z}{\partial x_{i}}\right) z e^{2 s \phi} d x
\end{aligned}
$$

Using known a priori estimates for elliptic equations (see [28]) we have

$$
\begin{aligned}
e^{s}\left\|\frac{\partial z\left(0, x^{\prime}\right)}{\partial x_{1}}\right\|_{W_{2}^{-\frac{1}{2}}(K)} & \leq C_{37} e^{s}\left(\|f\|_{L^{2}\left(-1,0 ; W_{2}^{-\frac{1}{2}}(K)\right)}+\|g\|_{W_{2}^{\frac{1}{2}}(K)}+\|z\|_{L^{2}(G)}\right) \\
& \leq C_{38}\left(\left\|f e^{s \phi}\right\|_{L^{2}\left(-1,0 ; W_{2}^{-\frac{1}{2}}(K)\right)}+\left\|g e^{s}\right\|_{W_{2}^{\frac{1}{2}}(K)}+\left\|z e^{s \phi}\right\|_{L^{2}(G)}\right) .
\end{aligned}
$$

From $(1.57)$ using $(1.56,1.58)$ and Cauchy-Bunyakovskii inequality we obtain

$$
\int_{G}\left(s^{-\frac{3}{4}}|\nabla z|^{2}+s^{\frac{5}{4}}|z|^{2}\right) e^{2 s \phi} d x \leq C_{39}\left(\|g\|_{W_{2}^{\frac{1}{2}}(K)}^{2} e^{2 s}+\int_{-1}^{0} s^{-\frac{1}{4}}\left\|f\left(x_{1}, \cdot\right)\right\|_{W_{2}^{-\frac{1}{2}}(K)}^{2} e^{2 s \phi} d x\right) .
$$

Let us introduce the function $\tilde{z}_{1}(x)=x_{1}^{10} z(x)$. By $(1.9,1.10)$ this function satisfies the equations

$$
\begin{gathered}
L \tilde{z}_{1}+\left[L, x_{1}^{10}\right] z=x_{1}^{10} f, \quad \tilde{z}_{1}\left(0, x^{\prime}\right)=\tilde{z}_{1}\left(-1, x^{\prime}\right)=0 \\
\tilde{z}_{1}\left(x_{1}, \ldots, x_{i}+2 \ell, . .\right)=\tilde{z}_{1}(x) \forall i \in\{2, \ldots, n\}, \quad \frac{\partial \tilde{z}_{1}\left(-1, x^{\prime}\right)}{\partial x_{1}}=0 .
\end{gathered}
$$

Obviously

Therefore, by (1.59)

$$
\left|\left[L, x_{1}^{10}\right] z(x)\right| \leq C_{40}\left(\left|x_{1}\right|^{9}|\nabla z(x)|+|z(x)|\right) \quad \forall x \in G
$$

$$
\begin{aligned}
\left\|\left[L, x_{1}^{10}\right] z e^{s \phi}\right\|_{L^{2}(G)}^{2} & \leq C_{41}\left(s^{-\frac{3}{8}}\left\||\nabla z| e^{s \phi}\right\|_{L^{2}(G)} s^{\frac{3}{8}}\left\|\left|\nabla \tilde{z}_{1}\right| e^{s \phi}\right\|_{L^{2}(G)}+\|z\|_{L^{2}(G)}^{2}\right) \\
& \leq C_{42}\left(s^{\frac{3}{4}}\left\|\left|\nabla \tilde{z}_{1}\right| e^{s \phi}\right\|_{L^{2}(G)}+\|g\|_{W_{2}^{\frac{1}{2}}(K)}^{2} e^{2 s}+\int_{-1}^{0} s^{-\frac{1}{4}}\left\|f\left(x_{1}, \cdot\right)\right\|_{W_{2}}^{2} e_{(K)}^{2 s \phi} d x\right) .
\end{aligned}
$$

Applying to $(1.60,1.61)$ the Carleman estimate from Proposition 1.1 with $\ell=0$ and using $(1.62)$ we obtain

$$
\int_{G}\left(s\left|\nabla \tilde{z}_{1}\right|^{2}+s^{3}\left|\tilde{z}_{1}\right|^{2}\right) e^{2 s \phi} d x \leq C_{43}\left(\int_{-1}^{0}\left\|f\left(x_{1}, \cdot\right)\right\|_{W_{2}^{-\frac{1}{2}}(K)}^{2} e^{2 s \phi} d x_{1}+\left\|x_{1}^{10} f e^{s \phi}\right\|_{L^{2}(G)}^{2}+\|g\|_{W_{2}^{\frac{1}{2}}(K)}^{2} e^{2 s}\right) .
$$


Hence estimate (1.49) follows from $(1.59,1.63)$. The proof of Lemma 1.4 is complete.

Let $\Gamma$ be an $(n-1)$ dimensional compact connected $C^{2}$ manifold without boundary in $\mathbb{R}^{n}$. We consider the following elliptic equation on the manifold $[-1,0] \times \Gamma$.

$$
\begin{gathered}
\tilde{A} z=-\sum_{i, j=0}^{n} \frac{\partial}{\partial x_{i}}\left(\tilde{a}_{i j}(\tilde{x}) \frac{\partial z}{\partial x_{j}}\right)+\sum_{i=0}^{n} \tilde{b}_{i}(\tilde{x}) \frac{\partial z}{\partial x_{i}}+\tilde{c}(\tilde{x}) z=f(\tilde{x}) \quad \text { in }[-1,0] \times \Gamma, \\
\left.z(0, \cdot)\right|_{\Gamma}=g(x),\left.\quad \frac{\partial z(-1, \cdot)}{\partial x_{0}}\right|_{\Gamma}=\left.z(-1, \cdot)\right|_{\Gamma}=0,
\end{gathered}
$$

where $\tilde{x}=\left(x_{0}, x\right)=\left(x_{0}, x_{1}, \ldots, x_{n}\right)$ and we assume that

$$
\tilde{a}_{i j} \in C^{2}([-1,0] \times \Gamma), \quad \tilde{b}_{i} \in C^{1}([-1,0] \times \Gamma), \quad \tilde{c} \in L^{\infty}([-1,0] \times \Gamma) .
$$

We have:

Lemma 1.5. Let the operator $\tilde{A}$ be elliptic on the manifold $[-1,0] \times \Gamma$, condition (1.66) be fulfilled and the function $\phi$ be defined as in (1.11). Then there exists a number $\hat{\lambda}>0$ such that for an arbitrary $\lambda \geq \hat{\lambda}$ there exists $s_{0}(\lambda)$ such that for each $s \geq s_{0}(\lambda)$ the solutions of problem (1.64-1.66) satisfy the following inequality:

$$
\begin{aligned}
\int_{[-1,0] \times \Gamma}\left(\left(x_{0}^{20} s^{\frac{3}{4}}\right.\right. & \left.\left.+s^{-\frac{3}{4}}\right)|\nabla z|^{2}+s^{\frac{5}{4}}|z|^{2}\right) e^{2 s \phi\left(x_{0}\right)} d x+s^{\frac{1}{4}} \int_{-1}^{0}\left\|\frac{\partial}{\partial x_{0}} z\left(x_{0}, \cdot\right)\right\|_{W_{2}^{-\frac{1}{2}}(\Gamma)}^{2} e^{2 s \phi\left(x_{0}\right)} d x_{0} \\
& \leq C_{25}\left(\int_{\Gamma} g^{2} e^{2 s \phi(0)} d \sigma+\int_{-1}^{0} s^{-\frac{1}{4}}\left\|f\left(x_{0}, \cdot\right)\right\|_{W_{2}}^{2}\left(\frac{1}{2}(\Gamma)\right.\right.
\end{aligned}
$$

where constant $C_{25}$ is independent of $s$.

Proof. Let $\left\{U_{k}\right\}_{k=1}^{K}$ be a covering of the manifold $\Gamma, h_{k}$ - are the local coordinates which corresponds to this system, and $\left\{e_{k}\right\}_{k=1}^{K}$ is the partition of unity such that $e_{k} \in C_{0}^{2}\left(U_{k}\right), \sum_{i=1}^{K} e_{k}(x)=1$ for all $x \in \Gamma$. Using the atlas $\left\{U_{k}, h_{k}\right\}$ we can introduce the new atlas $\left\{\tilde{U}_{k}, \tilde{h}_{k}\right\}$ on the manifold $[-1,0] \times \Gamma$ as follows $\tilde{U}_{k}=[-1,0] \times U_{k}, \tilde{h}_{k}=$ $\left(\tilde{h}_{1}^{(k)}, \ldots \tilde{h}_{n+1}^{(k)}\right)$ where $\tilde{h}_{1}^{(k)}=x_{0}, \tilde{h}_{2}^{(k)}=h_{1}^{(k)}(x), \ldots, \tilde{h}_{n+1}^{(k)}=h_{n}^{(k)}(x)$. Obviously the partition of unity $\left\{e_{k}\right\}_{k=1}^{K}$ has the same properties on manifold $[-1,0] \times \Gamma$ as on manifold $\Gamma: e_{k} \in C^{2}\left(\tilde{U}_{k}\right), \operatorname{supp} e_{k} \subset[-1,0] \times U_{k}, \sum_{i=1}^{k} e_{k}(x)=1$ for all $x \in[-1,0] \times U_{k}$. Also

$$
\left\|\left[\tilde{A}, e_{k}\right] z\left(x_{0}, \cdot\right)\right\|_{W_{2}^{-\frac{1}{2}}(\Gamma)} \leq C_{26}\left(\left\|z\left(x_{0}, \cdot\right)\right\|_{W_{2}^{\frac{1}{2}}(\Gamma)}+\left\|\frac{\partial z}{\partial x_{0}}\left(x_{0}, \cdot\right)\right\|_{W_{2}^{-\frac{1}{2}}(\Gamma)}\right)
$$

where the constant $C_{26}$ is independent of $x_{0}$. Denote $z_{k}=z e_{k}$. Then by $(1.64,1.65)$ function $z_{k}$ satisfies to equations

$$
\begin{gathered}
\tilde{A} z_{k}+\left[\tilde{A}, e_{k}\right] z=f e_{k} \quad \text { in }[-1,0] \times \Gamma \\
\left.z_{k}(0, \cdot)\right|_{\Gamma}=e_{k} g,\left.\quad \frac{\partial z_{k}(-1, \cdot)}{\partial x_{0}}\right|_{\Gamma}=\left.z_{k}(-1, \cdot)\right|_{\Gamma}=0 .
\end{gathered}
$$


In the local coordinates $\tilde{h}_{k}$ the function $\tilde{z}_{k}=z_{k}\left(\tilde{h}_{k}^{-1}(x)\right)$ satisfies to equations

$$
\begin{gathered}
-\sum_{i, j=1}^{n} \frac{\partial}{\partial x_{i}}\left(\tilde{a}_{i j}(x) \frac{\partial \tilde{z}_{k}}{\partial x_{j}}\right)+\sum_{i=1}^{n} \tilde{b}_{i}(x) \frac{\partial \tilde{z}_{k}}{\partial x_{i}}+\tilde{c}(x) \tilde{z}_{k}=\left(f e_{k}-\left[\tilde{A}, e_{k}\right] z\right) \circ \tilde{h}_{k}^{-1} \quad \text { in }[-1,0] \times h_{k}\left(U_{k}\right), \\
\left.\tilde{z}_{k}\right|_{[-1,0] \times \partial h_{k}\left(U_{k}\right)}=\left.\nabla \tilde{z}_{k}\right|_{[-1,0] \times \partial h_{k}\left(U_{k}\right)}=0, \quad \tilde{z}_{k}(0, \cdot)=g e_{k}, \quad \frac{\partial \tilde{z}_{k}(-1, \cdot)}{\partial x_{0}}=\tilde{z}_{k}(-1, \cdot)=0 .
\end{gathered}
$$

Taking sufficiently large cube $K=\prod_{i=1}^{n-1}[-\ell, \ell]$ we may assume that $h_{k}\left(U_{k}\right) \subset K$. Then by extending the functions $\tilde{z}_{k}, g e_{k} \circ h_{k}^{-1},\left(f e_{k}-\left[\tilde{A}, e_{k}\right] z\right) \circ h_{k}^{-1}$ by zero on the whole cube $K$, and the coefficients $\tilde{a}_{i j}, \tilde{b}_{i}, \tilde{c}$ up to periodic functions in $K$ by taking into account properties of regularity (1.4) and ellipticity (1.5) we obtain that the function $\tilde{z}_{k}$ satisfies to problem $(1.1-1.3)$ where $f=\left(f e_{k}-\left[\tilde{A}, e_{k}\right] z\right) \circ \tilde{h}_{k}^{-1}, g=g e_{k} \circ \tilde{h}_{k}^{-1}$ for all $k \in\{1, \ldots, K\}$. Thus by Lemma 1.4 and estimate (1.68) we have

$$
\begin{aligned}
& \int_{[-1,0] \times \Gamma}\left(\left(x_{0}^{20} s^{\frac{3}{4}}+s^{-\frac{3}{4}}\right)\left|\nabla z_{k}\right|^{2}+s^{\frac{5}{4}}\left|z_{k}\right|^{2}\right) e^{2 s \phi\left(x_{0}\right)} d \tilde{x}+s^{\frac{1}{4}} \int_{-1}^{0}\left\|\frac{\partial z_{k}}{\partial x_{0}}\left(x_{0}, \cdot\right)\right\|_{W_{2}^{-\frac{1}{2}}(\Gamma)}^{2} e^{2 s \phi\left(x_{0}\right)} d x_{0} \\
& \leq C_{27}\left(\|g\|_{W_{2}^{\frac{1}{2}}(\Gamma)}^{2} e^{2 s}+\int_{-1}^{0} s^{-\frac{1}{4}}\left(\left\|f\left(x_{0}, \cdot\right)\right\|_{W_{2}^{-\frac{1}{2}}(\Gamma)}^{2}+\left\|\frac{\partial z}{\partial x_{0}}\left(x_{0}, \cdot\right)\right\|_{W_{2}^{-\frac{1}{2}}(\Gamma)}^{2}+\left\|z\left(x_{0}, \cdot\right)\right\|_{W_{2}^{\frac{1}{2}}(\Gamma)}^{2}\right) e^{2 s \phi\left(x_{0}\right)} d x_{0}\right. \\
& \left.\quad+s^{-\frac{1}{4}} \int_{G} x_{1}^{20}\left(|\nabla z|^{2}+|f|^{2}\right) e^{2 s \phi\left(x_{0}\right)} d x\right), \forall s \geq s_{0}(k, \lambda) .
\end{aligned}
$$

Summing up inequalities (1.71) respect to the index $k$ we have

$$
\begin{aligned}
& \int_{[-1,0] \times \Gamma}\left(\left(x_{0}^{20} s^{\frac{3}{4}}+s^{-\frac{3}{4}}\right)|\nabla z|^{2}+s^{\frac{5}{4}}|z|^{2}\right) e^{2 s \phi\left(x_{0}\right)} d \tilde{x} \\
& +s^{\frac{1}{4}} \int_{-1}^{0}\left\|\frac{\partial z}{\partial x_{0}}\left(x_{0}, \cdot\right)\right\|_{W_{2}^{-\frac{1}{2}}(\Gamma)}^{2} e^{2 s \phi\left(x_{0}\right)} d x_{0} \\
& \leq C_{28}\left(\|g\|_{W_{2}^{\frac{1}{2}}(\Gamma)}^{2} e^{2 s \phi(0)}+\int_{-1}^{0} s^{-\frac{1}{4}\left(\left\|f\left(x_{0}, \cdot\right)\right\|_{W_{2}^{-\frac{1}{2}}(\Gamma)}^{2}\right.}\right. \\
& \left.+\left\|\frac{\partial z}{\partial x_{0}}\left(x_{0}, \cdot\right)\right\|_{W_{2}^{-\frac{1}{2}}(\Gamma)}^{2}+\left\|z\left(x_{0}, \cdot\right)\right\|_{W_{2}^{\frac{1}{2}}(\Gamma)}^{2}\right) e^{2 s \phi\left(x_{0}\right)} d x_{0} \\
& \left.\quad+s^{-\frac{1}{4}} \int_{G} x_{1}^{20}\left(|\nabla z|^{2}+|f|^{2}\right) e^{2 s \phi\left(x_{0}\right)} d x\right), \forall s \geq s_{0}=\max _{k} s_{0}(k, \lambda) .
\end{aligned}
$$

Thus, taking parameter $s_{0}$ sufficiently large for all $s \geq s_{0}$ we obtain

$$
\begin{aligned}
\int_{[-1,0] \times \Gamma}\left(\left(x_{0}^{20} s^{\frac{3}{4}}+s^{-\frac{3}{4}}\right)|\nabla z|^{2}+s^{\frac{5}{4}}|z|^{2}\right) e^{2 s \phi\left(x_{0}\right)} d x & \\
+s^{\frac{1}{4}} \int_{-1}^{0} \| & \frac{\partial}{\partial x_{0}} z\left(x_{0}, \cdot\right) \|_{W_{2}^{-1}(\Gamma)}^{2} e^{2 s \phi\left(x_{0}\right)} d x_{0} \leq C_{29}\left(\left\|g^{2} e^{s \phi(0)}\right\|_{W_{2}^{\frac{1}{2}}(\Gamma)}^{2}\right. \\
& \left.+\int_{-1}^{0} s^{-\frac{1}{4}}\left\|f\left(x_{0}, \cdot\right)\right\|_{W_{2}^{-\frac{1}{2}}(\Gamma)}^{2} e^{2 s \phi\left(x_{0}\right)} d x_{0}+s^{-\frac{1}{4}} \int_{G} x_{1}^{20}|f|^{2} e^{2 s \phi\left(x_{0}\right)} d x\right) .
\end{aligned}
$$

The proof of the lemma is finished. 
To obtain the Carleman estimate (1.7) by means of cutoff functions we divide the problems in two cases. In the first case the support of a function $y$ is concentrated near the boundary, and in the second case when $y$ has a compact support in $\Omega$. In order to deal with the first case we are going to use the Carleman estimate (1.67). Problem $(1.1,1.2)$ will become $(1.64,1.65)$ after a special change of variables which existence established in the following lemma:

Lemma 1.6. Under Condition 1 there exists $\varepsilon^{*}>0$ such that the set $\Omega_{\varepsilon}^{*}=\left\{x \mid 0 \leq \psi(x) \leq \varepsilon^{*}\right\}$ is $C^{2}$ diffeomorphic to the manifold $[-1,0] \times \Gamma$.

Proof. First we note that by Condition 1 there exists $\varepsilon_{0}$ sufficiently small such, that for all $\varepsilon \in\left(0, \varepsilon_{0}\right)$ the set $\Omega_{\varepsilon}=\{x \mid 0 \leq \psi(x) \leq \varepsilon\}$ consists of $N$ connected components $\Omega_{\varepsilon}^{i}$ such that $\Omega_{\varepsilon}^{i} \cap \Omega_{\varepsilon}^{j}=\{\emptyset\}$ for $i \neq j$. We assume that the set $\Omega_{\varepsilon}^{i}$ contains $\Gamma_{i}$. To prove this lemma it suffices to establish the existence of diffeomorphism of $\Omega_{\varepsilon}^{i}$ on the manifold $[-\varepsilon, 0] \times \Gamma_{i}$ for some $\varepsilon>0$. We construct a diffeomorphism $\eta(x)=(-\psi(x), \rho(x))$ from the set $\Omega_{\varepsilon}^{i}$ into $[-\varepsilon, 0] \times \Gamma_{i}$ where $\rho(x)=u$ is the solution to the extremal problem

$$
J(y)=\frac{1}{2}\|x-y\|^{2} \rightarrow \inf , \quad \psi(y)=0
$$

Let us prove that for all $x \in \Omega_{\varepsilon}^{i}$ with $\varepsilon$ sufficiently small this problem has only one solution. First, the existence of at least one solution $u \in \Gamma_{i}$ could be proved using standard arguments (see [25]). Applying the Lagrange principle to problem (1.73) we have

$$
x-u=\lambda \nabla \psi(u)
$$

where the Lagrange multiplier $\lambda \in \mathbb{R}^{1}$ is some constant. Thus, by (1.5)

$$
x-u=\|x-u\| \nabla \psi(u) /|\nabla \psi(u)|
$$

Suppose that for some $x \in \Omega_{\varepsilon}^{i}$ problem (1.73) has two solutions $u_{i}$. Obviously $\left\|x-u_{1}\right\|=\left\|x-u_{2}\right\|$, thus by (1.75)

$$
u_{2}-u_{1}=\left\|x-u_{1}\right\|\left(\nabla \psi\left(u_{1}\right) /\left|\nabla \psi\left(u_{1}\right)\right|-\nabla \psi\left(u_{2}\right) /\left|\nabla \psi\left(u_{2}\right)\right|\right)
$$

From $(1.5,1.75)$ we have

$$
\left\|u_{2}-u_{1}\right\| \leq\left\|x-u_{1}\right\| \hat{C}\left\|u_{2}-u_{1}\right\|
$$

where constant $\hat{C}$ only depends on the norm $\|\psi\|_{C^{2}(\bar{\Omega})}$. Thus, taking $\varepsilon_{0}$ small enough such that $\max _{x \in \Omega_{\varepsilon}^{i}}$ $\operatorname{dist}\left(x, \Gamma_{i}\right) \leq \frac{1}{\hat{C}+1}$ we obtain from (1.77) that $u_{1}=u_{2}$.

One can easily check, that for all sufficiently small $\varepsilon \eta\left(\Omega_{\varepsilon}^{i}\right) \subset[-\varepsilon, 0] \times \Gamma_{i}$. Let us show that the mapping $\eta$ is the one-to-one mapping. Our proof is by contradiction. Suppose that there exists the sequence $\left\{x_{k}^{(1)}, x_{k}^{(2)}\right\}_{k=1}^{\infty} \subset \mathbb{R}^{n}$ such that $\eta\left(x_{k}^{(1)}\right)=\eta\left(x_{k}^{(2)}\right)$ with $x_{k}^{(j)} \in \Omega_{1}^{i}$ and $x_{k}^{(1)} \neq x_{k}^{(2)}$. Without loss of generality one can assume that $x_{k}^{(j)} \rightarrow \tilde{x} \in \Gamma_{i}$. The equality $\eta\left(x_{k}^{(1)}\right)=\eta\left(x_{k}^{(2)}\right)$ implies $\psi\left(x_{k}^{(1)}\right)=\psi\left(x_{k}^{(2)}\right)$. Thus by considering the restriction of the function $\psi(x)$ on the line orthogonal to $\Gamma^{i}$ at the point $\rho\left(x_{k}^{(1)}\right)$ there exists at least one point $x_{k}^{(3)} \in\left[x_{k}^{(1)}, x_{k}^{(2)}\right]$ such that $\frac{\partial \psi\left(x_{k}^{(3)}\right)}{\partial \nu\left(\rho\left(x_{k}^{(1)}\right)\right)}=0$. Hence $\frac{\partial \psi(\tilde{x})}{\partial \nu(\tilde{x})}=0$ and $\nabla \psi(\tilde{x})=0$. But this contradicts to (1.5).

Obviously the mapping $\eta(x)$ is continuous. Our aim is to prove that $\eta \in C^{2}\left(\Omega_{\varepsilon}^{i} ;[-\varepsilon, 0] \times \Gamma_{i}\right)$. This statement is trivial for the first component of the vector-function $\eta$. Hence now we should prove $\rho \in C^{2}\left(\Omega_{\varepsilon}^{i} ;[-\varepsilon, 0] \times \Gamma_{i}\right)$. Let $\hat{x}$ be an arbitrary fixed point in $\Omega_{\varepsilon}^{i}$, and $\mathcal{U}$ be a sufficiently small neighborhood of this point in $\mathbb{R}^{n}$ and 
$\theta(u): B_{r}(0) \subset \mathbb{R}^{n-1} \rightarrow \Gamma_{i}$ the local coordinate system on the manifold $\Gamma_{i}$ in the neighborhood of $\rho(\hat{x}):$ $\rho(\hat{x}) \in \theta\left(B_{r}(0)\right)$. Then if $\rho(\hat{x})=\theta(\hat{v})$, the point $\hat{v}$ is the solution to the extremal problem

$$
J_{1}(v)=\|\hat{x}-\theta(v)\| \rightarrow \inf , \quad v \in B_{r}(0)
$$

and by the Fermat theorem the pair $(\hat{x}, \hat{v})$ satisfies to the system of linear equations

$$
\sum_{i=1}^{n}\left(\hat{x}_{i}-\theta_{i}(\hat{v})\right) \frac{\partial \theta_{i}(\hat{v})}{\partial v_{\ell}}=0 \quad \ell \in\{1, \ldots, n-1\}
$$

Moreover there exists an open set $\tilde{U} \subset U$ such that for all $x \in \tilde{U}$ exists $v \in B_{r}(0)$ that $\rho(x)=\theta(v)$ and equations $(1.78)$ hold true with $(x, v)$ exchanged for $(\hat{x}, \hat{v})$.

We introduce the new mapping

$$
F(x, v): \mathbb{R}^{n} \times \mathbb{R}^{n-1} \rightarrow \mathbb{R}^{n-1},
$$

where $F(x, v)=\left(F_{1}(x, v), \ldots, F_{n-1}(x, v)\right)$ and

$$
F_{\ell}(x, v)=\sum_{i=1}^{n}\left(x_{i}-\theta_{i}(v)\right) \frac{\partial \theta_{i}(v)}{\partial v_{\ell}} .
$$

Note that $\frac{\partial F(x, v)}{\partial v}$ is an epimorphism for all $v \in B_{r}(0)$ and $x \in \mathcal{U}$. In fact

$$
\frac{\partial F_{\ell}}{\partial v_{j}}(x, v)=-\sum_{i=1}^{n} \frac{\partial \theta_{j}(v)}{\partial v_{j}} \frac{\partial \theta_{i}(v)}{\partial v_{\ell}}-\sum_{i=1}^{n}\left(x_{i}-\theta_{i}(v)\right) \frac{\partial^{2} \theta_{i}(v)}{\partial v_{j} \partial v_{\ell}}
$$

So the matrix $\left\{\frac{\partial F_{\ell}}{\partial v_{j}}\right\}$ is the sum of two matrices $A(v)=\left\{a_{\ell j}\right\}=\left\{-\sum_{i=1}^{n} \frac{\partial \theta_{j}(v)}{\partial v_{j}} \frac{\partial \theta_{i}(v)}{\partial v_{\ell}}\right\}$ and $B(v)=\left\{b_{\ell j}\right\}=$ $\left\{-\sum_{i=1}^{n}\left(x_{i}-\theta_{i}(v)\right) \frac{\partial^{2} \theta_{i}(v)}{\partial v_{j} \partial v_{\ell}}\right\}$. For each $\delta>0$ there exists $\varepsilon_{0}>0$ such that for all $\varepsilon \in\left(0, \varepsilon_{0}\right)$

$$
\|B(v)\| \leq \delta .
$$

On the other hand rank $A=n-1$. Thus taking $\varepsilon_{0}$ sufficiently small we have

$$
\operatorname{rank}\left\{\frac{\partial F_{\ell}}{\partial v_{j}}\right\}=n-1
$$

Hence by implicit function theorem there exists a unique mapping $v=\mu(x)$ of class $C^{2}$ such that $F(x, \mu(x))=0$.

On the other hand, since by Condition 1 the manifold $\Gamma_{i}$ is compact one can choose $\varepsilon_{0}>0$ such that $\eta \in C^{2}\left(\Omega_{\varepsilon}^{i},[-\varepsilon, 0] \times \Gamma_{i}\right)$ for all $x \in \Omega_{\varepsilon}^{i}$ with $\varepsilon \in\left(0, \varepsilon_{0}\right)$.

Finally let us show that $\eta\left(\Omega_{\varepsilon}^{i}\right)=[-\varepsilon, 0] \times \Gamma_{i}$ for all $\varepsilon$ sufficiently small. Our proof is by contradiction. Suppose that there exists a sequence $\left\{y_{k}\right\}_{1}^{\infty}$ such that $y_{k} \in\left[-\frac{1}{k}, 0\right] \times \Gamma_{i}$ and $y_{k}=\left(y_{0, k}, y_{k}^{\prime}\right) \notin \eta\left(\Omega_{\frac{1}{k}}^{i}\right)$. Without loss of generality we can assume that $y_{k}^{\prime} \rightarrow \tilde{y}^{\prime}$. On the other hand on the line orthogonal to $\Gamma_{i}$ at the point $y_{k}^{\prime}$ one can find $\hat{x}_{k} \in \Omega_{\frac{1}{k}}^{i}$ such that $\psi\left(\hat{x}_{k}\right)=y_{0, k}$. But in this case equality (1.74) holds true with $x, u$ changed for $\hat{x}_{k}, y_{k}^{\prime}$. Moreover for $k$ sufficiently large one can choose the local coordinate system $\theta(u)$ on the manifold $\Gamma_{i}$ in the neighborhood of $y_{k}^{\prime}$ such that $\rho\left(x_{k}\right) \in \theta\left(B_{r}(0)\right), \tilde{y}^{\prime}=\theta(0)$. Let $y_{k}^{\prime}=\theta\left(v_{k}^{\prime}\right)$ and $\rho\left(\hat{x}_{k}\right)=\theta\left(\hat{v}_{k}\right)$. Then $v_{k}^{\prime} \rightarrow 0$ and $\hat{v}_{k} \rightarrow 0$. Thus $F\left(\hat{x}_{k}, \hat{v}_{k}\right)=F\left(\hat{x}_{k}, v_{k}^{\prime}\right)=0$. But in the small neighborhood of $\left(\tilde{y}^{\prime}, 0\right)$ this mapping has only one solution. We arrived to contradiction.

Now we are ready to prove Theorem 1.2.

Proof of Theorem 1.2. Condition 1 implies that for $\varepsilon$ sufficiently small the set $\Omega_{\varepsilon}=\{x \mid 0 \leq \psi(x) \leq \varepsilon\}$ consists of $N$ connected components. By Lemma 1.6 one can choose $\varepsilon^{*}$ small enough so that each component is 
diffeomorphic to manifold $[-1,0] \times \Gamma_{i}$. Hence there exists diffeomorphism $\eta(x): \Omega_{\varepsilon^{*}} \rightarrow[-1,0] \times \Gamma$ of class $C^{2}$. Let $e_{i}(x) i \in\{1,2\}$ be a set of functions such that $e_{1}(x) \in C^{2}(\bar{\Omega}), e_{2}(x) \in C_{0}^{2}\left(\overline{\Omega \backslash \Omega_{\varepsilon^{*} / 4}}\right), e_{1}(x)=1$ for all $x \in \Omega_{3 \varepsilon^{*} / 4}$, and $e_{1}(x)=0$ in $\Omega \backslash \Omega_{7 \varepsilon^{*} / 8}, e_{2}(x)=1$ for all $x \in \Omega \backslash \Omega_{\varepsilon^{*} / 2}$ and $e_{i}(x) \geq 0$ in $\Omega$. Obviously $|y(x)| \leq\left|y_{1}(x)\right|+\left|y_{2}(x)\right|$, where $y_{i}(x)=y(x) e_{i}(x)$. By (1.1-1.3) we have

$$
A y_{i}+\left[A, e_{i}\right] y=f e_{i} \quad \text { in } \Omega,\left.\quad y_{i}\right|_{\partial \Omega}=g e_{i}
$$

where $\left[A, e_{i}\right]$ is a first order differential operator with coefficients in $L^{\infty}(\Omega)$. It is well known (see [29], p. 102) that the diffeomorphism $\eta$ transfers the elliptic operator $A$ into the elliptic operator on manifold $[-1,0] \times \partial \Omega$ of the form

$$
\tilde{A}=\frac{\partial^{2}}{\partial x_{0}^{2}}+\sum_{j=0}^{1} A_{j}\left(x_{0}, x\right) \frac{\partial^{j}}{\partial x_{0}^{j}},
$$

where $A_{j}\left(x_{0}, x\right)$ is a differential operator of order $2-j$ on $\partial \Omega$.

Hence the function $\tilde{y}=y_{1} \circ \eta^{-1}$ is the solution to the boundary value problem

$$
\begin{gathered}
\tilde{A} \tilde{y}=\left(f e_{i}-\left[A, e_{i}\right] y\right) \circ \eta^{-1} \quad \text { in }[-1,0] \times \partial \Omega, \\
\left.\tilde{y}\right|_{x_{0}=0}=\left(g e_{1}\right) \circ \eta^{-1}, \quad \tilde{y}(-1, \cdot)=\frac{\partial \tilde{y}}{\partial x_{0}}(-1, \cdot)=0 .
\end{gathered}
$$

Thus, by Lemma 1.5 the estimate (1.67) for the function $\tilde{y}$ holds true. Moreover, it follows from the definition of the functions $e_{i}$, that all coefficients of the commutator $\left[A, e_{i}\right]$ have a compact support in $\Omega$. Thus by (1.1-1.3)

$$
\begin{gathered}
\sum_{|\alpha| \leq 1} \int_{\Omega}\left(s^{\frac{5-8|\alpha|}{4}}+|\psi|^{20|\alpha|}\right)\left|D^{\alpha} y\right|^{2} d x \leq \sum_{i=1}^{2} \sum_{|\alpha| \leq 1} \int_{\Omega}\left(s^{\frac{5-8|\alpha|}{4}}+|\psi|^{20|\alpha|}\right)\left|D^{\alpha} y_{i}\right|^{2} d x \\
\leq C_{31}\left(\sum_{i=1}^{2} \int_{\partial \Omega}\left|g e_{i}\right|^{2} e^{2 s \varphi} d \sigma+s^{-\frac{1}{4}} \int_{\Omega}\left(\left|f e_{i}\right|^{2}+\left|\left[A, e_{i}\right] y\right|^{2}\right) e^{2 s \varphi} d x\right. \\
\left.+\int_{\omega_{1}} s^{2} y^{2} e^{2 s \varphi} d x\right) \leq C_{32}\left(\|g\|_{W_{2}^{\frac{1}{2}}(\partial \Omega)}^{2} e^{2 s}+s^{-\frac{1}{4}} \int_{\Omega}\left(|f|^{2}+\psi^{10}|\nabla y|^{2}+y^{2}\right) e^{2 s \varphi} d x+\int_{\omega_{1}} s^{2} y^{2} e^{2 s \varphi} d x\right) \quad \forall s \geq s_{0},
\end{gathered}
$$

where we used Theorem 1.1 in order to estimate $y_{2}$. This inequality imply immediately Carleman estimate (1.7).

\section{Carleman estimate for the Stokes system}

In this section we will solve the observability problem for the linearized Navier-Stokes system. First let us consider the system of partial differential equations obtained from differential operator adjoint to the linearized Navier-Stokes system at point $\hat{v}$ by the change of variables $t \rightarrow-t$.

$$
\begin{gathered}
\frac{\partial y}{\partial t}-\Delta y+B^{*}(y, \hat{v})+B^{*}(\hat{v}, y)=\nabla p+f \text { in } Q \\
\operatorname{div} y=0,\left.\quad y\right|_{\Sigma}=0, \quad y(0, \cdot)=y_{0}
\end{gathered}
$$

where the operators $B^{*}(\cdot, \hat{v}), B^{*}(\hat{v}, \cdot)$ are defined by formulas

$$
B^{*}(y, \hat{v})=\left(\left(y, \frac{\partial \hat{v}}{\partial x_{1}}\right), \ldots,\left(y, \frac{\partial \hat{v}}{\partial x_{n}}\right)\right), \quad B^{*}(\hat{v}, y)=-(\hat{v}, \nabla) y
$$


We have:

Lemma 2.1. Let $\hat{v} \in W_{\infty}^{1}\left(0, T ;\left(V^{1}(\Omega) \bigcap\left(W_{\infty}^{1}(\Omega)\right)^{n}\right)\right)$. Then for each $y_{0} \in V^{1}(\Omega), f \in L^{2}\left(0, T ; V^{0}(\Omega)\right)$ there exists a unique solution $y \in V^{1,2}(Q)$ to problem (2.1, 2.2). Moreover this solution satisfies to the estimate

$$
\|y\|_{V^{1,2}(Q)} \leq C_{1}\left(\left\|y_{0}\right\|_{V^{1}(\Omega)}+\|f\|_{L^{2}\left(0, T ; V^{0}(\Omega)\right)}\right) .
$$

This lemma can be proved by standard arguments (see for example [31]). In order to formulate our Carleman estimate let us first introduce the weight functions

$$
\alpha(t, x)=\frac{e^{\lambda \psi(x)}-e^{\lambda^{2}\|\psi\|_{C(\bar{\Omega})}}}{(t(T-t))^{8}}, \hat{\alpha}(t)=\left.\alpha(t, x)\right|_{\partial \Omega}=\frac{1-e^{\lambda^{2}\|\psi\|_{C(\bar{\Omega})}}}{(t(T-t))^{8}}, \hat{\varphi}(t)=\frac{1}{(t(T-t))^{8}}
$$

where $\lambda>1$ is some parameter to be fixed below. By $(1.5,2.4)$

$$
\hat{\alpha}(t) \leq \alpha(t, x) \quad \forall(t, x) \in Q
$$

First let us remind some standard Carleman estimates for the heat equation

$$
\frac{\partial z}{\partial t}-\Delta z=f \text { in } Q,\left.\quad z\right|_{\Sigma}=0
$$

We have:

Lemma 2.2 [20]. Let $z \in L^{2}(Q)$ be solution to (2.6) and $f \in L^{2}(Q)$. Then there exists a $\hat{\lambda}>1$ such that for any $\lambda>\hat{\lambda}$ one can find $s_{0}(\lambda)$ such that the following inequality holds

$$
\begin{aligned}
\int_{Q}\left(\frac{1}{s \hat{\varphi}}\left(\left|\frac{\partial z}{\partial t}\right|^{2}+\sum_{i, j=1}^{n}\left|\frac{\partial^{2} z}{\partial x_{i} \partial x_{j}}\right|^{2}\right)+s \hat{\varphi}|\nabla z|^{2}+s^{3} \hat{\varphi}^{3} z^{2}\right) e^{2 s \alpha} d x d t & \\
& \leq C_{2}\left(\int_{Q} f^{2} e^{2 s \alpha} d x d t+\int_{Q_{\omega_{1}}} s^{3} \hat{\varphi}^{3} z^{2} e^{2 s \alpha} d x d t\right) \forall s \geq s_{0}
\end{aligned}
$$

where the constant $C_{2}$ is independent of $s$ and $\omega_{1}$ is the subdomain from the Lemma 1.1.

Now we can prove the following theorem:

Theorem 2.1. Let $y \in L^{2}\left(0, T ; V^{0}(\Omega)\right)$ be solution to (2.1, 2.2) and $f \in L^{2}\left(0, T ; V^{0}(\Omega)\right)$. Then there exists a $\hat{\lambda}>1$ such that for any $\lambda>\hat{\lambda}$ one can find $s_{0}(\lambda)$ such that the following inequality holds

$$
\begin{aligned}
I(s)=\int_{Q}\left(\frac{1}{s \hat{\varphi}}\left(\left|\frac{\partial y}{\partial t}\right|^{2}+\sum_{i, j=1}^{n}\left|\frac{\partial^{2} y}{\partial x_{i} \partial x_{j}}\right|^{2}\right)+s \hat{\varphi}|\nabla y|^{2}+s^{3} \hat{\varphi}^{3} y^{2}\right) e^{2 s \alpha} d x d t \\
\leq C_{3}\left(\int_{Q} f^{2}\left(e^{2 s \alpha}+(s \hat{\varphi})^{\frac{3}{4}} e^{2 s \hat{\alpha}}\right) d x d t+\int_{Q_{\omega_{1}}}\left(s^{3} \hat{\varphi}^{3} y^{2}+(s \hat{\varphi})^{\frac{11}{4}} p^{2}\right) e^{2 s \alpha} d x d t\right) \forall s \geq s_{0}
\end{aligned}
$$

where the constant $C_{3}$ is independent of $s$ and $\omega_{1}$ is the subdomain from the Lemma 1.1.

Proof. Applying the Carleman estimate (2.7) to the linearized Navier-Stokes system and keeping in mind the inequality

$$
\left\|B^{*}(y(t, \cdot), \hat{v}(t, \cdot))\right\|_{\left(L^{2}(\Omega)\right)^{n}}^{2}+\left\|B^{*}(\hat{v}(t, \cdot), y(t, \cdot))\right\|_{\left(L^{2}(\Omega)\right)^{n}}^{2} \leq C_{4} \int_{\Omega}\left(|\nabla y(t, x)|^{2}+|y(t, x)|^{2}\right) d x
$$


we have

$$
I(s) \leq C_{5}\left(\int_{Q_{\omega_{1}}} s^{3} \hat{\varphi}^{3} y^{2} e^{2 s \alpha} d x d t+\int_{Q}|\nabla p|^{2} e^{2 s \alpha} d x d t+\int_{Q} f^{2} e^{2 s \alpha} d x d t\right) \forall s \geq s_{0}(\lambda) .
$$

Applying the divergence operator div to both parts of equation (2.1) we obtain

$$
\Delta p(t, \cdot)=\operatorname{div}\left(B^{*}(\hat{v}(t, \cdot), y(t, \cdot))+B^{*}(y(t, \cdot), \hat{v}(t, \cdot))\right) \text { in } \Omega
$$

for a.e. $t \in[0, T]$.

Note that

$$
\left|\operatorname{div}\left(B^{*}(\hat{v}(t, x), y(t, x))+B^{*}(y(t, x), \hat{v}(t, x))\right)\right| \leq C_{6}(|\nabla y(t, x)|+|y(t, x)|) \quad \forall(t, x) \in Q .
$$

From $(2.10)$, using the estimates $(1.7,2.11)$ we can estimate the gradient of the pressure as follows

$$
\begin{aligned}
\int_{\Omega}|\nabla p(t, \cdot)|^{2} e^{2 s \alpha} d x \leq C_{7}\left(\|p(t, \cdot)\|_{W_{2}^{\frac{1}{2}}(\partial \Omega)}^{2}(s \hat{\varphi}(t))^{\frac{3}{4}} e^{2 s \hat{\alpha}(t)}\right. & \\
& \left.+\int_{\Omega}(s \hat{\varphi})^{\frac{1}{2}}\left(|\nabla y|^{2}+|y|^{2}\right) e^{2 s \alpha} d x+\int_{\omega_{1}}(s \hat{\varphi})^{\frac{11}{4}} p^{2} e^{2 s \alpha} d x\right),
\end{aligned}
$$

where before applying (1.7) we made the change $s \rightarrow s /(t(T-t))^{8}$ and we multiplied the inequality (1.7) by $\exp \left\{-\exp \left\{\lambda^{2}\|\psi\|_{C(\bar{\Omega})}\right\} /(t(T-t))^{8}\right\}$. Inequalities $(2.9,2.12)$ imply

$$
\begin{aligned}
I(s) \leq & C_{8}\left(\int_{Q_{\omega_{1}}}\left(s^{3} \hat{\varphi}^{3} y^{2}+(s \hat{\varphi})^{\frac{11}{4}} p^{2}\right) e^{2 s \alpha} d x d t\right. \\
& \left.+\int_{0}^{T}(s \hat{\varphi}(t))^{\frac{3}{4}}\|p(t, \cdot)\|_{W_{2}^{\frac{1}{2}}(\partial \Omega)}^{2} e^{2 s \hat{\alpha}} d t+\int_{Q} f^{2} e^{2 s \alpha} d x d t\right) \forall s \geq s_{0}(\lambda) .
\end{aligned}
$$

To estimate the norm of the trace of the pressure on the boundary we introduce the new function $w(t, x)=$ $y(t, x) \hat{\varphi}^{\frac{3}{8}}(t) e^{s \hat{\alpha}(t)}$. By $(2.1,2.2)$ this function satisfies to the system of equations

$$
\begin{aligned}
L w-\left(s \hat{\alpha}_{t}+\frac{\partial \hat{\varphi}^{\frac{3}{8}}}{\partial t} \hat{\varphi}^{-\frac{3}{8}}\right) w & =\nabla p \hat{\varphi}^{\frac{3}{8}} e^{s \hat{\alpha}}+f \hat{\varphi}^{\frac{3}{8}} e^{s \hat{\alpha}} \quad \text { in } \Omega, \\
\left.w\right|_{\Sigma}=0, \operatorname{div} w & =0, w(0, \cdot)=0 .
\end{aligned}
$$

By the Sobolev embedding Theorem and the standard energy a priori estimates for solutions of $(2.14,2.15)$ we have

$$
\begin{aligned}
\int_{0}^{T}\|p(t, \cdot)\|_{W_{2}^{\frac{1}{2}}(\partial \Omega)}^{2}(s \hat{\varphi})^{\frac{3}{4}} e^{2 s \hat{\alpha}} d t \leq C_{9} \int_{Q}\left(|\nabla p|^{2}+p^{2}\right)(s \hat{\varphi})^{\frac{3}{4}} e^{2 s \hat{\alpha}} d x d t \\
\quad \leq C_{10}\left(\int_{Q}\left((s \hat{\varphi})^{\frac{3}{4}}\left|s \hat{\alpha}_{t}+\frac{\partial \hat{\varphi} \frac{3}{8}}{\partial t} \hat{\varphi}^{-\frac{3}{8}}\right|^{2} y^{2} e^{2 s \hat{\alpha}}+|f|^{2}(s \varphi)^{\frac{3}{4}} e^{2 s \hat{\alpha}}\right) d x d t+\int_{Q_{\omega}} p^{2}(s \hat{\varphi})^{\frac{3}{4}} e^{2 s \hat{\alpha}} d x d t\right) .
\end{aligned}
$$

Taking into account that

$$
\hat{\varphi}^{\frac{3}{4}}(t)\left(\left|\hat{\alpha}_{t}(t)\right|^{2}+\left|\frac{\partial \hat{\varphi}^{\frac{3}{8}}(t)}{\partial t} \hat{\varphi}^{-\frac{3}{8}}(t)\right|^{2}\right) \leq C_{11} \hat{\varphi}^{3}(t) \quad \forall t \in[0, T]
$$


we obtain from the previous inequality

$$
\begin{aligned}
\int_{0}^{T}(s \hat{\varphi})^{\frac{3}{4}}\|p(t, \cdot)\|_{W_{2}^{\frac{1}{2}}(\partial \Omega)}^{2} e^{2 s \hat{\alpha}} d t \leq & C_{12}\left(\int_{0}^{T}(s \hat{\varphi})^{\frac{3}{4}}\|p(t, \cdot)\|_{L^{2}\left(\omega_{1}\right)}^{2} e^{2 s \hat{\alpha}} d t\right. \\
& \left.+\int_{Q}\left(s^{\frac{11}{4}} \hat{\varphi}^{3} y^{2} e^{2 s \alpha}+|f|^{2}(s \hat{\varphi})^{\frac{3}{4}} e^{2 s \hat{\alpha}}\right) d x d t\right) .
\end{aligned}
$$

From $(2.5,2.13,2.16)$ the Carleman estimate (2.8) follows immediately.

Let us consider the system of partial differential equations which is obtained from $(2.1,2.2)$ by change of variables $t \rightarrow-t$ :

$$
\begin{gathered}
L^{*} z=-\frac{\partial z}{\partial t}-\Delta z+B^{*}(z, \hat{v})+B^{*}(\hat{v}, z)=\nabla q+f \text { in } Q \\
\operatorname{div} z=0,\left.\quad z\right|_{\Sigma}=0, \quad z(T, \cdot)=z_{0}
\end{gathered}
$$

where the operators $B^{*}(\hat{v}, \cdot), B^{*}(\cdot, \hat{v})$ are defined in (2.3). A short calculation shows, that $L^{*}$ is the adjoint of the differential operator which corresponds to the linearization of the Navier-Stokes equations at the point $\hat{v}$.

We need the following technical lemma:

Lemma 2.3. Let $z_{0} \in V^{0}(\Omega)$ and $\hat{v} \in W_{\infty}^{1}\left(0, T ;\left(V^{1}(\Omega) \cap\left(W_{\infty}^{1}(\Omega)\right)^{n}\right)\right.$. Then the solutions of problem (2.17, 2.18) satisfy the estimate

$$
\left\|\frac{\partial z}{\partial t}\right\|_{L^{2}\left(0, T / 2 ; V^{0}(\Omega)\right)}+\|z\|_{L^{2}\left(0, T / 2 ; V^{2}(\Omega)\right)} \leq C_{13}\left(\|z\|_{L^{2}\left(0,3 T / 4 ; V^{1}(\Omega)\right)}+\|f\|_{L^{2}\left(0,3 T / 4 ; V^{0}(\Omega)\right)}\right) .
$$

The proof of this lemma is given in Appendix.

Let us consider the boundary value problem for the stationary Stokes system

$$
\Delta v=\nabla q+g \text { in } \Omega, \operatorname{div} v=0,\left.v\right|_{\partial \Omega}=0
$$

The following lemma is proved in [31]:

Lemma 2.4. For any $g \in V^{-1}(\Omega)$ there exists a unique solution $v \in V^{1}(\Omega)$ of problem (2.20) and this solution satisfies the estimate

$$
\|v\|_{V^{1}(\Omega)} \leq C_{14}\|g\|_{V^{-1}(\Omega)} .
$$

Let us introduce the function $\kappa(t, x)$ by the formula

$$
\begin{gathered}
\kappa(t, x)=\left(e^{\tilde{\lambda} \psi}-e^{\tilde{\lambda}^{2}\|\psi\|_{C(\bar{\Omega})}}\right) /(\ell(t)(T-t))^{8}, \hat{\kappa}(t)=\min _{x \in \bar{\Omega}} \kappa(t, x), \quad \tilde{\kappa}(t)=\max _{x \in \bar{\Omega}} \kappa(t, x), \\
\ell \in C^{\infty}[0, T], \quad \ell(t)=t \forall t \in[3 T / 2, T], \quad \ell(t)>0 \quad \forall t \in[0, T] .
\end{gathered}
$$

Let us take the parameter $\tilde{\lambda}$ such that

$$
\tilde{\lambda}>\hat{\lambda}
$$

where $\hat{\lambda}$ defined in Theorem 2.1 and

$$
\max _{x \in \bar{\Omega}} \kappa(t, x)<\frac{9}{10} \min _{x \in \bar{\Omega}} \kappa(t, x) \quad \forall t \in[0, T] .
$$


Note that

$$
\kappa(t, x)=\alpha_{\tilde{\lambda}}(t, x) \quad \forall(t, x) \in\left(\frac{3}{2} T, T\right) \times \Omega .
$$

Now we are ready to prove an observability inequality for system $(2.17,2.18)$. We have

Theorem 2.2. Let pair $(z, q) \in V^{1,2}(Q) \times L^{2}\left(0, T ; W_{2}^{1}(\Omega)\right)$ satisfies (2.17, 2.18), $f \in L^{2}\left(0, T ; V^{0}(\Omega)\right)$. Then there exists $\hat{s}>0$ such that inequality holds

$$
\|z(0, \cdot)\|_{V^{0}(\Omega)}^{2}+\int_{Q}(T-t)^{8}|z|^{2} e^{\hat{s} \kappa} d x d t \leq C_{15}\left(\int_{Q_{\omega}}|z|^{2} e^{\frac{9}{10} \hat{s} \hat{\kappa}} d x d t+\int_{Q}|f|^{2} e^{\frac{9}{10} \hat{s} \hat{\kappa}} d x d t\right)
$$

where the constant $C_{15}$ is independent of $f, z$.

Proof. Let us introduce the functions $r, g, \tilde{f}$ by formulas

$$
r(t, x)=\int_{\frac{T}{2}}^{t} z(\tau, x) d \tau, \quad g(t, x)=\int_{\frac{T}{2}}^{t} q(\tau, x) d \tau, \quad \tilde{f}(t, x)=\int_{\frac{T}{2}}^{t} f(\tau, x) d \tau .
$$

Short calculations shows that the pair $(r, g)$ satisfies the equations

$$
\begin{gathered}
L^{*} r=\nabla g+\int_{\frac{T}{2}}^{t} B^{*}\left(r(s, \cdot), \frac{\partial \hat{v}(s, \cdot)}{\partial s}\right) d s+\int_{\frac{T}{2}}^{t} B^{*}\left(\frac{\partial \hat{v}(s, \cdot)}{\partial s}, r(s, \cdot)\right) d s-z\left(\frac{T}{2}, \cdot\right)+\tilde{f} \text { in } Q \\
\operatorname{div} r=0,\left.\quad r\right|_{\Sigma}=0 .
\end{gathered}
$$

Let us show that the function $g$ satisfies the estimate

$$
\begin{aligned}
\|g(t, \cdot)\|_{L^{2}\left(\omega_{1}\right)} \leq & C_{16}\left(\left\|z\left(\frac{T}{2}, \cdot\right)\right\|_{\left(L^{2}(\omega)\right)^{n}}+\|z\|_{\left(L^{2}\left(\left(\frac{T}{2}, t\right) \times \omega\right)\right)^{n}}+\|z(t, \cdot)\|_{\left(L^{2}(\omega)\right)^{n}}\right. \\
& \left.+\|r(t, \cdot)\|_{\left(L^{2}(\omega)\right)^{n}}+\|\tilde{f}(t, \cdot)\|_{\left(L^{2}(\omega)\right)^{n}}\right)
\end{aligned}
$$

where $C_{16}$ is independent of $t \in[0, T]$. Using the definition of the function $r$ we can rewrite equation (2.26) as follows

$$
\begin{aligned}
-\Delta r=\nabla g-z\left(\frac{T}{2}, \cdot\right)+z(t, \cdot) & -B^{*}(r, \hat{v})-B^{*}(\hat{v}, r) \\
& +\int_{\frac{T}{2}}^{t} B^{*}\left(r(s, \cdot), \frac{\partial \hat{v}(s, \cdot)}{\partial s}\right) d s+\int_{\frac{T}{2}}^{t} B^{*}\left(\frac{\partial \hat{v}(s, \cdot)}{\partial s}, r(s, \cdot)\right) d s+\tilde{f} \quad \text { in } \Omega
\end{aligned}
$$

Note that the function $g$ in $(2.29)$ is defined up to a constant. To fix it we set

$$
\int_{\omega_{1}} q(t, x) d x=0 \quad \forall t \in[0, T]
$$

This equality implies

$$
\int_{\omega_{1}} g(t, x) d x=0 \quad \forall t \in[0, T]
$$


We are looking for the functions $r, g$ in the form $r=r_{1}+r_{2}, g=g_{1}+g_{2}$, where

$$
\begin{aligned}
-\Delta r_{1}=\nabla g_{1}-z( & \left.\frac{T}{2}, \cdot\right)+z(t, \cdot)-B^{*}(r, \hat{v})-B^{*}(\hat{v}, r) \\
& +\int_{\frac{T}{2}}^{t} B^{*}\left(r(s, \cdot), \frac{\partial \hat{v}(s, \cdot)}{\partial s}\right) d s+\int_{\frac{T}{2}}^{t} B^{*}\left(\frac{\partial \hat{v}(s, \cdot)}{\partial s}, r(s, \cdot)\right) d s+\tilde{f} \text { in } \omega \\
& \operatorname{div} r_{1}=0,\left.r_{1}\right|_{\partial \omega}=0, \int_{\omega_{1}} g_{1}(t, x) d x=0 t \in(0, T) .
\end{aligned}
$$

By Lemma 2.4 the unique solution of problem (2.31) exists and satisfies the estimate

$$
\begin{aligned}
\left\|r_{1}\right\|_{V^{1}(\omega)}+\left\|g_{1}\right\|_{L^{2}(\omega)} \leq C_{17}\left(\left\|z\left(\frac{T}{2}, \cdot\right)\right\|_{\left(L^{2}(\omega)\right)^{n}}+\|z(t, \cdot)\|_{\left(L^{2}(\omega)\right)^{n}}\right. \\
\left.+\|r\|_{\left(L^{2}\left(\left(\frac{T}{2}, t\right) \times \omega\right)\right)^{n}}+\|r(t, \cdot)\|_{\left(L^{2}(\omega)\right)^{n}}+\|\tilde{f}(t, \cdot)\|_{\left(L^{2}(\omega)\right)^{n}}\right)
\end{aligned}
$$

By virtue of $(2.29,2.27,2.31)$ the functions $r_{2}, q_{2}$ should satisfy the equations

$$
\Delta r_{2}=\nabla g_{2} \text { in } \omega, \operatorname{div} r_{2}=0
$$

Thus

$$
\Delta g_{2}=0 \text { in } \omega
$$

Applying the Laplace operator $\Delta$ to this last equation we obtain $\Delta^{2} r_{2}=0$. Thus thanks to (2.33) and from well known estimates about interior regularity of solutions of elliptic equations (see [24]) we have

$$
\begin{aligned}
\left\|r_{2}(t)\right\|_{\left(C^{2}\left(\bar{\omega}_{1}\right)\right)^{n}} \leq & C_{18}\left\|r(t)-r_{1}(t)\right\|_{\left(L^{2}(\omega)\right)^{n}} \leq C_{19}\left(\left\|z\left(\frac{T}{2}, \cdot\right)\right\|_{\left(L^{2}(\omega)\right)^{n}}\right. \\
& \left.+\|r\|_{\left(L^{2}\left(\left(\frac{T}{2}, t\right) \times \omega\right)\right)^{n}}+\|r(t, \cdot)\|_{\left(L^{2}(\omega)\right)^{n}}+\|z(t, \cdot)\|_{\left(L^{2}(\omega)\right)^{n}}+\|\tilde{f}(t, \cdot)\|_{\left(L^{2}(\omega)\right)^{n}}\right) .
\end{aligned}
$$

By (2.34) equality (2.33) implies the estimate

$$
\begin{aligned}
\left\|\nabla g_{2}(t, \cdot)\right\|_{\left(C\left(\bar{\omega}_{1}\right)\right)^{n}} \leq & C_{20}\left(\left\|z\left(\frac{T}{2}, \cdot\right)\right\|_{\left(L^{2}(\omega)\right)^{n}}+\|r(t, \cdot)\|_{\left(L^{2}(\omega)\right)^{n}}\right. \\
& \left.+\|r\|_{\left(L^{2}\left(\left(\frac{T}{2}, t\right) \times \omega\right)\right)^{n}}+\|\tilde{f}(t, \cdot)\|_{\left(L^{2}(\omega)\right)^{n}}+\|z(t, \cdot)\|_{\left(L^{2}(\omega)\right)^{n}}\right) .
\end{aligned}
$$

By $(2.30,2.31)$ we obtain

$$
\int_{\omega_{1}} g_{2}(t, x) d x=0 \forall t \in[0, T] .
$$


Thus inequality (2.35) yields

$$
\begin{aligned}
\left\|g_{2}(t, \cdot)\right\|_{L^{2}\left(\omega_{1}\right)} \leq & C_{21}\left(\left\|z\left(\frac{T}{2}, \cdot\right)\right\|_{\left(L^{2}(\omega)\right)^{n}}+\|r(t, \cdot)\|_{\left(L^{2}(\omega)\right)^{n}}+\|r\|_{\left(L^{2}\left(\left(\frac{T}{2}, t\right) \times \omega\right)\right)^{n}}\right. \\
& \left.+\|\tilde{f}(t, \cdot)\|_{\left(L^{2}(\omega)\right)^{n}}+\|z(t, \cdot)\|_{\left(L^{2}(\omega)\right)^{n}}\right) .
\end{aligned}
$$

This inequality and (2.32) imply (2.28).

Note that, since for each fixed $x$ the function $\alpha(t, x)$ reaches it maximum at $T / 2$ we have

$$
\int_{Q}\left|\int_{\frac{T}{2}}^{t} B^{*}\left(r(s, \cdot), \frac{\partial \hat{v}(s, \cdot)}{\partial s}\right) d s+\int_{\frac{T}{2}}^{t} B^{*}\left(\frac{\partial \hat{v}(s, \cdot)}{\partial s}, r(s, \cdot)\right) d s\right|^{2} e^{2 s \alpha} d x d t \leq C_{22} \int_{Q}\left(|\nabla r|^{2}+r^{2}\right) e^{2 s \alpha} d x d t
$$

where the constant $C_{22}$ is independent of $s$.

Applying Carleman inequality (2.8) to equations $(2.26,2.27)$ and taking into account (2.37) we have

$$
\begin{aligned}
\int_{Q}\left(\frac { 1 } { s \hat { \varphi } } \left(|z|^{2}+\right.\right. & \left.\left.\sum_{i, j=1}^{n}\left|\frac{\partial^{2} r}{\partial x_{i} \partial x_{j}}\right|^{2}\right)+s \hat{\varphi}|\nabla r|^{2}+s^{3} \hat{\varphi}^{3}|r|^{2}\right) e^{2 s \alpha} d x d t \\
\leq & C_{23}(\tilde{\lambda})\left(\int_{Q_{\omega_{1}}}(s \varphi)^{\frac{11}{4}} g^{2} e^{2 s \alpha} d x d t+\int_{Q_{\omega_{1}}} s^{3} \hat{\varphi}^{3}|r|^{2} e^{2 s \alpha} d x d t\right. \\
& \left.+\int_{Q}(s \hat{\varphi})^{\frac{3}{4}}\left(|\tilde{f}|^{2}+\left|z\left(\frac{T}{2}, x\right)\right|^{2}\right) e^{2 s \alpha} d x d t\right)
\end{aligned}
$$

where $s \geq s_{0}(\tilde{\lambda})$.

Parameter $s_{0}(\tilde{\lambda})$ is defined in Theorem 2.1. Set $\hat{s}=2 s_{0}(\tilde{\lambda})$. Using a priori estimate (2.19) for system $(2.17$, 2.18 ) in the right hand side of inequality (2.38) we can replace the function $\alpha$ by $\kappa$ and the function $\tilde{\varphi}$ by $(T-t)^{-8}$ and the constant $C_{23}$ by $C_{24}(s)$ :

$$
\begin{aligned}
\int_{Q}(T-t)^{8}|z|^{2} e^{s \kappa} d x d t & +\left\|z\left(\frac{T}{2}, \cdot\right)\right\|_{V^{1}(\Omega)}^{2} \leq C_{24}(s)\left(\int_{Q_{\omega_{1}}} \frac{g^{2}}{(T-t)^{22}} e^{s \kappa} d x d t\right. \\
& \left.+\int_{Q_{\omega_{1}}} \frac{|r|^{2}}{(T-t)^{24}} e^{s \kappa} d x d t+\int_{Q} \frac{1}{(T-t)^{6}}|\tilde{f}|^{2} e^{s \kappa} d x d t+\left\|z\left(\frac{T}{2}, \cdot\right)\right\|_{V^{0}(\Omega)}^{2}\right)
\end{aligned}
$$

where $s \geq \hat{s}$.

Using estimate (2.28) one can rewrite (2.39) as follows

$$
\begin{aligned}
\int_{Q}(T-t)^{8}|z|^{2} e^{\hat{s} \kappa} d x d t & +\left\|z\left(\frac{T}{2}, \cdot\right)\right\|_{V^{1}(\Omega)}^{2} \leq C_{25}\left(\int_{Q_{\omega_{1}}}(T-t)^{-24}|r|^{2} e^{\hat{s} \tilde{\kappa}} d x d t\right. \\
& +\int_{Q_{\omega}} \frac{\left(|\tilde{f}|^{2}+|z|^{2}+|r|^{2}\right)}{(T-t)^{24}} e^{\hat{s} \tilde{\kappa}} d x d t+\int_{0}^{T} \frac{\|z\|_{\left.\left(L^{2}\left(\left(\frac{T}{2}, t\right) \times \omega\right)\right)\right)^{n}}^{2}}{(T-t)^{24} \tilde{\hat{\kappa}}} d t \\
& \left.+\int_{Q} \frac{1}{(T-t)^{6}}|\tilde{f}|^{2} e^{\hat{s} \kappa} d x d t+\left\|z\left(\frac{T}{2}, \cdot\right)\right\|_{V^{0}(\Omega)}^{2}\right) .
\end{aligned}
$$


Note that by $(2.22-2.24)$

$$
\begin{aligned}
\int_{Q_{\omega}} \frac{\left(|\tilde{f}|^{2}+|z|^{2}+|r|^{2}\right)}{(T-t)^{24}} e^{\hat{s} \tilde{\kappa}} d x d t & +\int_{Q} \frac{|\tilde{f}|^{2}}{(T-t)^{6}} e^{\hat{s} \kappa} d x d t \\
& +\int_{0}^{T} \frac{\|z\|_{\left.\left(L^{2}\left(\left(\frac{T}{2}, t\right) \times \omega\right)\right)\right)^{n}}^{2}}{(T-t)^{24}} e^{\hat{s} \tilde{\kappa}} d t \leq C_{26}\left(\int_{Q}|f|^{2} e^{\frac{9}{10} \hat{s} \hat{\kappa}} d x d t+\int_{Q_{\omega}}|z|^{2} e^{\frac{9}{10} \hat{s} \hat{\kappa}} d x d t\right)
\end{aligned}
$$

where $C_{26}$ is independent constant. Thanks to this last inequality we deduce from (2.40)

$$
\begin{aligned}
\int_{Q}|z|^{2}(T-t)^{8} e^{\hat{s} \kappa} d x d t & +\left\|z\left(\frac{T}{2}, \cdot\right)\right\|_{V^{1}(\Omega)}^{2} \leq C_{27}\left(\int_{Q_{\omega}}|z|^{2} e^{\frac{9}{10} \hat{s} \hat{\kappa}} d x d t\right. \\
& \left.+\int_{Q}|f|^{2} e^{\frac{9}{10} \hat{s} \hat{\kappa}} d x d t+\left\|z\left(\frac{T}{2}, \cdot\right)\right\|_{V^{0}(\Omega)}^{2}\right) .
\end{aligned}
$$

Let us finish the proof by contradiction. By Lemma 2.1 instead of showing the estimate (2.25) it suffices to prove

$$
\int_{Q}|z|^{2}(T-t)^{8} e^{\hat{s} \kappa} d x d t+\left\|z\left(\frac{T}{2}, \cdot\right)\right\|_{V^{1}(\Omega)}^{2} \leq C_{28}\left(\int_{Q_{\omega}}|z|^{2} e^{\frac{9}{10} \hat{s} \hat{\kappa}} d x d t+\int_{Q}|f|^{2} e^{\frac{9}{10} \hat{s} \hat{\kappa}} d x d t\right)
$$

If inequality (2.42) is not true, then by $(2.41)$ there exists a sequence $\left(z_{k}, q_{k}, f_{k}\right)$ such that

$$
L^{*} z_{k}=\nabla q_{k}+f_{k} \text { in } Q, \operatorname{div} z_{k}=0,\left.z_{k}\right|_{\Sigma}=0, \lim _{k \rightarrow \infty}\left\|z_{k}\left(\frac{T}{2}, \cdot\right)\right\|_{\left(L^{2}(\Omega)\right)^{n}}>0
$$

$$
\begin{aligned}
f_{k} \rightarrow 0 \text { in }\left(L^{2}\left(Q, e^{\frac{9}{10} \hat{s} \hat{\kappa}}\right)\right)^{n}, & \int_{Q_{\omega}}\left|z_{k}\right|^{2} e^{\frac{9}{10} \hat{s} \hat{\kappa}} d x d t \rightarrow 0 \text { as } k \rightarrow \infty \\
& z_{k}\left(\frac{T}{2}, \cdot\right) \rightarrow z\left(\frac{T}{2}, \cdot\right) \text { in }\left(L^{2}(\Omega)\right)^{n}, z_{k} \rightarrow z \text { weakly in } V^{1,2}((0, T-\epsilon) \times \Omega)
\end{aligned}
$$

for all $\epsilon \in(0, T)$. Passing to the limit in (2.43), and taking into account (2.44) we obtain

$$
\begin{gathered}
L^{*} z=\nabla q \text { in } Q, \quad \operatorname{div} z=0,\left.\quad z\right|_{\Sigma}=0,\left.\quad z\right|_{Q_{\omega}} \equiv 0, \\
\left\|z\left(\frac{T}{2}, \cdot\right)\right\|_{\left(L^{2}(\Omega)\right)^{n}} \neq 0 .
\end{gathered}
$$

From $(2.8,2.45)$ we obtain

$$
z \equiv 0
$$

but this is impossible by virtue of (2.46). Therefore the proof of lemma is complete. 


\section{Solvability of Linear Controllability PRoblem}

Let us consider the problem of exact controllability of the linearized Navier-Stokes equations:

$$
\begin{gathered}
L y=\frac{\partial y}{\partial t}-\Delta y+B(y, \hat{v})+B(\hat{v}, y)=\nabla p+f+\chi_{\omega} u \text { in } Q, \\
\operatorname{div} y=0,\left.\quad y\right|_{\Sigma}=0, \quad y(0, x)=y_{0}(x), \\
y(T, x)=0,
\end{gathered}
$$

where the functions $y_{0}, f$ are given and $u$ is a control taken in the space $\left(L^{2}\left(Q_{\omega}\right)\right)^{n}$. Before studying solvability of problem (3.1-3.3) let us recall some existence theorems for boundary value problem (3.1,3.2), assuming that $u$ is a fixed function.

Let us set

$$
\eta(t, x)=-\hat{s} \kappa(t, x)
$$

where the function $\kappa$ defined in (2.22) and the parameter $\hat{s}$ have been taken from Theorem 2.2. Since the function $\kappa(t, x)$ is negative, $\eta(t, x)$ is positive. Moreover $\lim _{t \rightarrow T-0} \eta(t, x)=+\infty$.

Later on we use the following weight function:

$$
\theta(t, x)=\left(1-\chi_{\omega}\right) \frac{e^{\eta}}{(T-t)^{8}}+\chi_{\omega} .
$$

To formulate our results we need to introduce some non-standard functional spaces

$$
\begin{aligned}
F(Q, \theta)=\left\{f \in\left(L_{2}(Q)\right)^{n}:\right. & \exists f_{1} \in\left(L_{2}(Q, \theta)\right)^{n}, \\
& \left.\exists f_{2} \in L_{2}\left(0, T ; W_{2}^{1}(\Omega)\right) \text { such that } f=f_{1}+\nabla f_{2}\right\} .
\end{aligned}
$$

The norm of the space $F(Q, \theta)$ is defined by the relation

$$
\|f\|_{F(Q, \theta)}=\inf _{\substack{f_{1}, \nabla f_{2} \\ f=f_{1}+\nabla f_{2}}}\left(\left\|f_{1}\right\|_{\left(L_{2}(Q, \theta)\right)^{n}}^{2}+\left\|\nabla f_{2}\right\|_{\left(L_{2}(Q)\right)^{n}}^{2}\right)^{1 / 2} .
$$

We are looking for solution of the controllability problem in the following space

$$
Y(Q)=\left\{y \in V^{1,2}(Q) \mid L y \in F(Q, \theta), e^{-\frac{2}{5} \hat{s} \hat{\kappa}} y \in V^{1,2}(Q)\right\}
$$

equipped with the norm

$$
\|y\|_{Y(Q)}^{2}=\|L y\|_{F(Q, \theta)}^{2}+\left\|e^{-\frac{2}{5} \hat{s} \hat{\kappa}} y\right\|_{V^{1,2}(Q)}^{2} .
$$

where the function $\kappa$ introduced in (2.22) and the operator $L$ determined in (3.1). Now we can convert observability inequality (2.25) into the controllability result for the linearized Navier-Stokes system (3.1-3.3). We have

Theorem 3.1. Let $f \in F(Q, \theta), y_{0} \in V^{1}(\Omega)$. Then there exists a solution of problem (3.1-3.3) $(y, p, u) \in$ $Y(Q) \times L^{2}\left(0, T ; W_{2}^{1}(\Omega)\right) \times\left(L^{2}\left(Q_{\omega}\right)\right)^{n}$ which satisfies the estimate

$$
\|(y, p, u)\|_{Y(Q) \times L^{2}\left(0, T ; W_{2}^{1}(\Omega)\right) \times\left(L^{2}\left(Q_{\omega}\right)\right)^{n}} \leq C_{1}\left(\left\|y_{0}\right\|_{V^{1}(\Omega)}+\|f\|_{F(Q, \theta)}\right) .
$$


Proof. We first assume that $f \in L^{2}(Q, \theta)$ and $\left.f\right|_{Q^{\omega}} \equiv 0$. Let us consider the extremal problem

$$
\begin{gathered}
\mathcal{J}_{k}(y, u)=\frac{1}{2} \int_{Q} \rho_{k}|y|^{2} d x d t+\frac{1}{2} \int_{Q} m_{k}|u|^{2} d x d t \rightarrow \text { inf }, \\
L y=u+\nabla p+f \quad \text { in } \quad Q, \operatorname{div} y=0,\left.y\right|_{\Sigma}=0, \quad y(0, x)=y_{0}(x), \quad y(T, x)=0,
\end{gathered}
$$

where

$$
\rho_{k}(t)=e^{\frac{-9 \hat{s} \hat{\kappa}(t)(T-t)^{8}}{10(T-t+1 / k)^{8}}}, \quad m_{k}(t, x)=\left\{\begin{array}{l}
e^{\frac{-9}{10} \frac{\hat{s} \hat{\kappa}(T-t)}{(T-t+1 / k)^{8}}}, x \in \bar{\omega} \\
k, x \in \Omega \backslash \bar{\omega}
\end{array}\right.
$$

Obviously the functions $\rho_{k}, m_{k}$ are bounded in $Q$ for every $k>0$.

By Lemma 2.1 the exists an admissible element to problem $(3.8,3.9)$. So it is easy to prove (see $[25,26])$ that problem $(3.8,3.9)$ has a unique solution, which we denote as $\left(\hat{y}_{k}, \hat{p}_{k}, \hat{u}_{k}\right) \in V^{1,2}(Q) \times L^{2}\left(0, T ; W_{2}^{1}(\Omega)\right) \times\left(L^{2}(Q)\right)^{n}$.

Thus, applying the Lagrange principle to extremal problem $(3.8,3.9)$ (see $[1,9]$ ) we obtain

$$
\begin{gathered}
L \hat{y}_{k}=f+\nabla p_{k}+\hat{u}_{k} \text { in } Q, \operatorname{div} \hat{y}_{k}=0,\left.\hat{y}_{k}\right|_{\Sigma}=0, \hat{y}_{k}(T, \cdot) \equiv 0, \hat{y}_{k}(0, \cdot)=y_{0}, \\
L^{*} z_{k}=\nabla q_{k}+\rho_{k} \hat{y}_{k} \text { in } Q,\left.\quad z_{k}\right|_{\Sigma}=0, \operatorname{div} z_{k}=0, z_{k}=-m_{k} \hat{u}_{k} \text { in } Q
\end{gathered}
$$

where the operator $L^{*}$ defined in (2.17) is an operator conjugate to the operator $L$.

Since the function $\rho_{k}$ only depends on the variable $t$ only, by Lemma $2.1 \rho_{k} \hat{y}_{k} \in L^{2}\left(0, T ; V^{1}(\Omega)\right)$. So we can apply the estimate (2.25) to equation (3.11) in order to obtain:

$$
\int_{Q}(T-t)^{8} e^{\hat{s} \kappa}\left|z_{k}\right|^{2} d x d t+\left\|z_{k}(0, \cdot)\right\|_{\left(L^{2}(\Omega)\right)^{n}}^{2} \leq C_{2}\left(\int_{Q} \rho_{k}^{2} e^{\frac{9}{10} \hat{s} \hat{\kappa}}\left|\hat{y}_{k}\right|^{2} d x d t+\int_{Q} e^{\frac{9}{10} \hat{s} \hat{\kappa}}\left|z_{k}\right|^{2} d x d t\right) .
$$

We observe that $\left|\rho_{k}(t) e^{\frac{9}{10} \hat{s} \hat{\kappa}(t)}\right| \leq 1$ for all $(t, x) \in Q$ and $\left|m_{k}(t, x) e^{\frac{9}{10} \hat{s} \hat{\kappa}(t)}\right| \leq 1$ for all $(t, x) \in Q_{\omega}$. In fact

$$
-\frac{9 \hat{s} \hat{\kappa}(t)(T-t)^{8}}{10(T-t+1 / k)^{8}}+\frac{9}{10} \hat{s} \hat{\kappa}(t)=-\frac{9}{10} \frac{\hat{s}}{\ell(t)} \max _{x \in \bar{\Omega}}\left(e^{\tilde{\lambda}^{2}\|\psi\|_{C(\bar{\Omega})}}-e^{\tilde{\lambda} \psi(x)}\right)\left(\frac{1}{(T-t)^{8}}-\frac{1}{(T-t+1 / k)^{8}}\right)<0 .
$$

Keeping in mind these inequalities and substituting the function $z_{k}$ by using the forth equation in (3.11) in the last integral of equality (3.12) we have

$$
\int_{\Omega}\left|z_{k}(0, x)\right|^{2} d x+\int_{Q}\left|z_{k}\right|^{2}(T-t)^{8} e^{\hat{s} \kappa} d x d t \leq C_{3}\left(\int_{Q} \rho_{k}\left|\hat{y}_{k}\right|^{2} d x d t+\int_{Q_{\omega}} m_{k}\left|\hat{u}_{k}\right|^{2} d x d t\right) .
$$

Multiplying $\left(3.11_{1}\right)$ by $\hat{y}_{k}$ scalarly in $\left(L^{2}(Q)\right)^{n}$, and integrating by parts with respect to $t$ and $x$, bearing in mind (3.10) after simplifications we have

$$
\begin{aligned}
0 & =\left(L^{*} z_{k}-\nabla q_{k}-\rho_{k} \hat{y}_{k}, \hat{y}_{k}\right)_{\left(L^{2}(Q)\right)^{n}}=-\int_{Q} \rho_{k}\left|\hat{y}_{k}\right|^{2} d x d t+\left(z_{k}, L \hat{y}_{k}\right)_{\left(L^{2}(Q)\right)^{n}}+\left(z_{k}(0, \cdot), \hat{y}_{k}(0, \cdot)\right)_{\left(L^{2}(\Omega)\right)^{n}} \\
& =-\int_{Q} \rho_{k}\left|\hat{y}_{k}\right|^{2} d x d t-\int_{Q} m_{k}\left|\hat{u}_{k}\right|^{2} d x d t+\int_{Q}\left(f, z_{k}\right) d x d t+\left(z_{k}(0, \cdot), y_{0}\right)_{\left(L^{2}(\Omega)\right)^{n}}
\end{aligned}
$$

Hence

$$
\mathcal{J}_{k}\left(\hat{y}_{k}, \hat{u}_{k}\right)=\frac{1}{2} \int_{Q}\left(\rho_{k}\left|\hat{y}_{k}\right|^{2}+m_{k}\left|\hat{u}_{k}\right|^{2}\right) d x d t=\frac{1}{2}\left(\int_{Q}\left(f, z_{k}\right) d x d t+\left(z_{k}(0, \cdot), y_{0}\right)_{\left(L^{2}(\Omega)\right)^{n}}\right) .
$$


Note that

$$
\left|\int_{Q}\left(f, z_{k}\right) d x d t\right| \leq C_{4}\|f\|_{\left(L^{2}(Q, \theta)\right)^{n}}\left\|(T-t)^{4} e^{\hat{s} \kappa / 2} z_{k}\right\|_{\left(L^{2}(Q)\right)^{n}} .
$$

By $(3.13-3.15)$ we obtain

$$
\mathcal{J}_{k}\left(\hat{y}_{k}, \hat{u}_{k}\right) \leq C_{5}\left(\|f\|_{F(Q, \theta)}+\left\|y_{0}\right\|_{\left(L^{2}(\Omega)\right)^{n}}\right) \sqrt{\mathcal{J}_{k}\left(\hat{y}_{k}, \hat{u}_{k}\right)}
$$

Hence

$$
\mathcal{J}_{k}\left(\hat{y}_{k}, \hat{u}_{k}\right) \leq C_{5}^{2}\left(\|f\|_{F(Q, \theta)}^{2}+\left\|y_{0}\right\|_{\left(L^{2}(\Omega)\right)^{n}}^{2}\right)
$$

By virtue of $(3.16,3.4)$ we have a subsequence $\left\{\left(\hat{y}_{k}, \hat{u}_{k}\right)\right\}_{k=1}^{\infty}$ such that

$$
\begin{aligned}
& \left(\hat{y}_{k}, \hat{u}_{k}\right) \rightarrow(y, u) \quad \text { weakly in } V^{1,2}(Q) \times\left(L^{2}(Q)\right)^{n}, \\
& \hat{u}_{k} \rightarrow 0 \text { in }\left(L^{2}((0, T) \times(\Omega \backslash \omega))\right)^{n}, \\
& \int_{Q_{\omega}} m_{k}\left|\hat{u}_{k}\right|^{2} d x d t+\int_{Q} \rho_{k}\left|\hat{y}_{k}\right|^{2} d x d t \leq C_{6} .
\end{aligned}
$$

Also, by $(3.13,3.17)$ it follows from $(3.11)$

$$
\left\|m_{k} \hat{u}_{k}\right\|_{V^{1,2}((0, T-\varepsilon) \times \Omega)} \leq C_{7}(\varepsilon)
$$

for all $\varepsilon \in(0, T)$. Hence without loss of generality one can assume

$$
\hat{u}_{k}(t, x) \rightarrow u(t, x) \quad \text { almost everywhere in } Q_{\omega} .
$$

Using (3.17), we pass to the limit in (3.10) to obtain that pair $(y, u)$ is a solution of problem (3.1-3.3). The relations $(3.16,3.17)$ and Lemma 2.1 imply the estimate

$$
\|(y, p, u)\|_{V^{1,2}(Q) \times L^{2}\left(0, T ; W_{2}^{1}(\Omega)\right) \times\left(L^{2}\left(Q_{\omega}\right)\right)^{n}} \leq C_{8}\left(\left\|y_{0}\right\|_{V^{1}(\Omega)}+\|f\|_{F(Q, \theta)}\right) .
$$

Also by $(3.17,3.18)$ and Fatou's theorem (see [23], p. 307) we have

$$
\|(y, u)\|_{\left(L^{2}\left(Q, e^{-\frac{9}{10} \hat{s} \hat{\kappa}}\right)\right)^{n} \times\left(L^{2}\left(Q_{\omega}, e^{-\frac{9}{10} \hat{s} \hat{\kappa}}\right)\right)^{n}} \leq C_{6} .
$$

Now to prove (3.7) we need only to estimate the norm of the function $e^{-\frac{2}{5} \hat{s} \hat{\kappa}} y$ in the space $V^{1,2}(Q)$. Let us make the following change of variables in (3.1). Set $\tilde{y}=y e^{-\frac{2}{5} \hat{s} \hat{\kappa}}, \tilde{p}=p e^{-\frac{2}{5} \hat{s} \hat{\kappa}}, g=\left(f-\frac{2}{5} \hat{s} \frac{\partial \hat{\kappa}}{\partial t} y\right) e^{-\frac{2}{5} \hat{s} \hat{\kappa}}, \tilde{u}=u e^{-\frac{2}{5} \hat{s} \hat{\kappa}}, \tilde{y}_{0}=$ $y_{0} e^{-\frac{2}{5} \hat{s} \hat{\kappa}(0)}$. Note that by $(3.19,3.20)$

$$
\|g\|_{\left(L^{2}(Q)\right)^{n}}+\|\tilde{u}\|_{\left(L^{2}(Q)\right)^{n}} \leq C_{9}\left(\left\|y_{0}\right\|_{V^{0}(\Omega)}+\|f\|_{F(Q, \theta)}\right) .
$$

We observe, that the pair $(\tilde{y}, \tilde{p})$ satisfies the equation

$$
\begin{gathered}
L \tilde{y}=\nabla \tilde{p}+g+\chi_{\omega} \tilde{u} \text { in } Q \\
\operatorname{div} \tilde{y}=0,\left.\quad \tilde{y}\right|_{\Sigma}=0, \tilde{y}(0, x)=\tilde{y}_{0}(x)
\end{gathered}
$$

Thus (3.19-3.21) and a priori estimate taken from Lemma 2.1 implies (3.7). 
Now let $f \in F(Q, \theta)$ be an arbitrary function. Then there exist functions $f_{1} \in\left(L^{2}(Q, \theta)\right)^{n}$ and $f_{2} \in$ $L^{2}\left(0, T ; W_{2}^{1}(\Omega)\right)$ such that $f=f_{1}+\nabla f_{2}$. Above we proved that there exists a solution $(y, p, u)$ of the exact controllability problem $(3.1-3.3)$ with initial datum $\left(y_{0},\left(1-\chi_{\omega}\right) f_{1}\right)$ which satisfy the estimate (3.7). Obviously $\left(y, p+f_{2}, u+\chi_{\omega} f_{1}\right)$ is a solution of this problem for the initial datum $\left(y_{0}, f\right)$.

\section{Proof of the MaIN theorem}

Now our nearest aim is to reduce the proof of Theorem 1 to the case of a linear controllability problem. We look for a solution of problem $(1,2,4,5)$ in the form

$$
v(t, x)=\hat{v}(t, x)+y(t, x)
$$

Substitution of (4.1) into equations $(1,2,5)$ and then subtraction from them of the same equation for $\hat{v}$ yields

$$
\begin{gathered}
\mathcal{N}(y, q, u)=\partial_{t} y(t, x)-\Delta y+B(\hat{v}, y)+B(y, \hat{v})+B(y, y)-\nabla q-\chi_{\omega} u=0 \text { in } \Omega, \\
\operatorname{div} y=0 \\
y(0, x)=v_{0}(x)-\hat{v}(0, x) \\
y(T, x)=0 .
\end{gathered}
$$

We will solve the problem (4.2-4.5) with help of the following variant of the implicit function Theorem (see [1]).

Theorem 4.1 (on a right inverse operator). Suppose that $X, Z$ are Banach spaces and

$$
\mathcal{A}: X \rightarrow Z
$$

is a continuously differentiable map. We assume that for $x_{0} \in X, z_{0} \in Z$ the equality

$$
\mathcal{A}\left(x_{0}\right)=z_{0}
$$

holds and the derivative $\mathcal{A}^{\prime}\left(x_{0}\right): X \rightarrow Z$ of the map $\mathcal{A}$ at $x_{0}$ is epimorphism. Then there exists $\epsilon>0$ such that for any $z \in Z$ which satisfies the condition

$$
\left\|z-z_{0}\right\|_{Z}<\epsilon
$$

there exists a solution $x \in X$ of the equation

$$
\mathcal{A}(x)=z
$$

In our case the space

$$
X=Y(Q) \times L^{2}\left(0, T ; W_{2}^{1}(\Omega)\right) \times\left(L^{2}\left(Q_{\omega}\right)\right)^{n}
$$

and

$$
Z=F(Q, \theta) \times V^{1}(\Omega) .
$$

The operator $\mathcal{A}$ is defined by the formula

$$
\mathcal{A}(y, q, u)=(\mathcal{N}(y, q, u), y(0, \cdot))
$$

We have:

Lemma 4.1. Let $\hat{v} \in W_{\infty}^{1}\left(0, T ; V^{1}(\Omega) \bigcap\left(W_{\infty}^{1}(\Omega)\right)^{n}\right)$, then $\mathcal{A} \in C^{1}(X, Y)$. 
Proof. It follows directly from the definitions of the spaces $X, Z$ that the operator

$$
(y, q, u) \rightarrow\left(\partial_{t} y(t, x)-\Delta y+B(\hat{v}, y)+B(y, \hat{v})-\nabla q-\chi_{\omega} u, y(0, \cdot)\right): X \rightarrow Z
$$

is continuous and moreover continuously differentiable in virtue of linearity. The operator $B$ is a bilinear one. So to prove this lemma it is sufficient to establish continuity of the bilinear operator

$$
B: Y(Q) \times Y(Q) \rightarrow\left(L^{2}(Q, \theta)\right)^{n}
$$

where the function $\theta$ was defined in (3.6). Note that from $(2.4,2.22,3.5)$

$$
\eta(t, x) \leq-\hat{s} \hat{\kappa}(t, x) \quad \forall(t, x) \in Q
$$

Then (4.10) and simple transformations give the estimate

$$
\begin{aligned}
\left\|B\left(y_{1}, y_{2}\right)\right\|_{\left(L^{2}(Q, \theta)\right)^{n} \leq}^{2} \leq & C_{1} \sum_{i, j=1}^{2}\left(\int_{Q \backslash Q_{\omega}} \frac{\left|y_{i}\right|^{2}\left|\nabla y_{j}\right|^{2} e^{\eta}}{(T-t)^{8}} d x d t\right. \\
& \left.+\int_{Q_{\omega}}\left|y_{i}\right|^{2}\left|\nabla y_{j}\right|^{2} d x d t\right) \leq C_{2} \sum_{i, j=1}^{2} \int_{Q} \frac{\left|y_{i}\right|^{2}\left|\nabla y_{j}\right|^{2}}{(T-t)^{8}} e^{-\hat{s} \hat{\kappa}} d x d t \\
\leq & C_{3}\left\|e^{-\frac{4}{5} \hat{s} \hat{\kappa}} y_{1}\right\|_{V^{1,2}(Q)}^{2}\left\|e^{-\frac{4}{5} \hat{s} \hat{\kappa}} y_{2}\right\|_{V^{1,2}(Q)}^{2} .
\end{aligned}
$$

This inequality proves the theorem.

Proof of the Theorem 1. Firstly we apply the inverse operator theorem to the problem (4.2-4.5). Let $\mathcal{A}$ be the operator defined by formulas $(4.9,4.2)$, and let the spaces $X, Z$ be defined as in $(4.7,4.8)$. Set $x_{0}=(0,0,0)$, $z_{0}=(0,0)$. Then equation (4.6) obviously holds. By Lemma $4.1 \mathcal{A} \in C^{1}(X, Z)$ and by Theorem $3.1 \operatorname{Im\mathcal {A}^{\prime }(0)=}$ $Z$. So all necessary conditions to apply the theorem about the inverse operator are fulfilled. Therefore there exists such $\varepsilon>0$ such that for any initial data (4.4) satisfying the inequality

$$
\left\|y_{0}\right\|_{V^{1}(\Omega)} \leq \varepsilon
$$

the problem $(4.2-4.5)$ has a solution $(y, p, u) \in X$. Then the triple $(y+\hat{v}, p+\hat{p}, u)$ is a solution of the problem $(1-$ $3,5)$.

\section{Appendix}

Proof of Lemma 2.3. Making the change of the variable $t \rightarrow(T-t)$ in $(2.17,2.18)$ we obtain the system

$$
\begin{gathered}
\frac{\partial z}{\partial t}-\Delta z+B^{*}(z, \mathbf{v})+B^{*}(\mathbf{v}, z)=\nabla q+\mathbf{f} \text { in } Q \\
\operatorname{div} z=0,\left.\quad z\right|_{\Sigma}=0, \quad z(0, \cdot)=z_{0}
\end{gathered}
$$

where $\mathbf{v}(t, x)=\hat{v}(T-t, x), \mathbf{f}(t, x)=f(T-t, x)$. Now instead of (2.19) it suffices to prove

$$
\left\|\frac{\partial z}{\partial t}\right\|_{L^{2}\left(T / 2, T ; V^{0}(\Omega)\right)}+\|z\|_{L^{2}\left(T / 2, T ; V^{2}(\Omega)\right)} \leq C\left(\|z\|_{L^{2}\left(T / 4, T ; V^{1}(\Omega)\right)}+\|\mathbf{f}\|_{L^{2}\left(T / 4, T ; V^{0}(\Omega)\right)}\right) .
$$


The Sobolev embedding theorem implies the following estimates

$$
\begin{aligned}
\left\|B^{*}(z, \mathbf{v})+B^{*}(\mathbf{v}, z)\right\|_{L^{2}\left(0, T ; V^{-1}(\Omega)\right)} & \leq C\|z\|_{L^{2}\left(0, T ; V^{0}(\Omega)\right)}\|\mathbf{v}\|_{L^{\infty}\left(0, T ;\left(W_{\infty}^{1}(\Omega)\right)^{n}\right)} \\
\left\|B^{*}(z, \mathbf{v})+B^{*}(\mathbf{v}, z)\right\|_{L^{2}\left(0, T ; V^{0}(\Omega)\right)} & \leq C\|z\|_{L^{2}\left(0, T ; V^{1}(\Omega)\right)}\|\mathbf{v}\|_{L^{\infty}\left(0, T ;\left(W_{\infty}^{1}(\Omega)\right)^{n}\right)}
\end{aligned}
$$

Thanks to inequalities (4) using the Faedo-Galerkin method and arguing exactly same way as in [31] (p. 255) we can construct a solution to problem $(1,2)$ such that

$$
s\left\|\frac{\partial z}{\partial t}\right\|_{L^{2}\left(0, T ; V^{0}(\Omega)\right)}+\|z\|_{L^{2}\left(0, T ; V^{1}(\Omega) \cap\left(W_{2}^{1+s}(\Omega)\right)^{n}\right)} \leq C\left(\left\|z_{0}\right\|_{V^{s}(\Omega)}+\|\mathbf{f}\|_{L^{2}\left(0, T ; V^{-1+s}(\Omega)\right)}\right) \quad s \in\{0,1\} .
$$

Thus for $z \in V^{0}(\Omega), \mathbf{f} \in L^{2}\left(0, T ; V^{0}(\Omega)\right)$ there exists a solution to problem $(1,2)$ a function $z \in L^{2}\left(0, T ; V^{1}(\Omega)\right)$. Denote $\mathbf{z}=\rho z$, where $\rho \in C^{\infty}[0, T], \rho(t)=1$ for all $t \in[T / 2, T]$ and $\rho(t)=0$ for all $t \in[0, T / 4]$. The function $\mathbf{z}$ satisfies to the system of equations

$$
\begin{gathered}
\frac{\partial \mathbf{z}}{\partial t}-\Delta \mathbf{z}+B^{*}(\mathbf{z}, \mathbf{v})+B^{*}(\mathbf{v}, \mathbf{z})=\nabla(\rho q)+z \frac{\partial \rho}{\partial t}+\rho \mathbf{f} \text { in } Q \\
\operatorname{div} \mathbf{z}=0,\left.\quad \mathbf{z}\right|_{\Sigma}=0, \quad \mathbf{z}(0, \cdot)=0
\end{gathered}
$$

Thus applying to problem $(6,7)$ the apriori estimate (5) with $s=0$ we obtain $(2.19)$. The proof of the lemma is complete.

The author wish to acknowledge the many helpfull comments and suggestions of referees that resulted in an improved paper.

\section{REFERENCES}

[1] V.M. Alekseev, V.M. Tikhomirov and S.V. Fomin, Optimal control. Consultants Bureau, New York (1987).

[2] D. Chae, O.Yu. Imanuvilov and S.M. Kim, Exact controllability for semilinear parabolic equations with Neumann boundary conditions. J. Dynam. Control Systems 2 (1996) 449-483.

[3] J.-M. Coron, On the controllability of the 2-D incompressible Navier-Stokes equations with the Navier-Slip boundary conditions. ESAIM: COCV 1 (1996) 35-75.

[4] J.-M. Coron, On the controllability of 2-D incompressible perfect fluids. J. Math. Pures Appl. 75 (1996) 155-188.

[5] J.-M. Coron, Contrôlabilité exacte frontière de l'équation d'Euler des fluides parfaits incompressibles bidimensionnels. $C$. $R$. Acad. Sci. Paris Sér. I Math. 317 (1993) 271-276.

[6] J.-M. Coron and A.V. Fursikov, Global exact controllability of the 2-D Navier-Stokes equations on manifold without boundary. Russian J. Math. Phys. 4 (1996) 1-20.

[7] C. Fabre, Résultats d'unicité pour les équations de Stokes et applications au contrôle. C. R. Acad. Sci. Paris Sér. I Math. 322 (1996) 1191-1196.

[8] C. Fabre and G. Lebeau, Prolongement unique des solutions de l'équation de Stokes. Comm. Partial Differential Equations 21 (1996) 573-596.

[9] A.V. Fursikov and O.Yu. Imanuvilov, Local exact controllability of two dimensional Navier-Stokes system with control on the part of the boundary. Sb. Math. 187 (1996) 1355-1390.

[10] A.V. Fursikov and O.Yu. Imanuvilov, Local exact boundary controllability of the Boussinesq equation. SIAM J. Control Optim. 36 (1988) 391-421.

[11] A.V. Fursikov and O.Yu. Imanuvilov, Local exact controllability of the Navier-Stokes Equations. C. R. Acad. Sci. Paris Sér. I Math. 323 (1996) 275-280.

[12] A.V. Fursikov and O.Yu. Imanuvilov, Controllability of evolution equations, Lecture notes series (1996), no. 34 SNU, Seoul.

[13] A.V. Fursikov and O.Yu. Imanuvilov, On approximate controllability of the Stokes system. Ann. Fac. Sci. Toulouse 11 (1993) 205-232.

[14] A.V. Fursikov and O.Yu. Imanuvilov, Exact controllability of the Navier-Stokes equations and the Boussinesq system. Russian Math. Surveys 54 (1999) 565-618.

[15] O. Glass, Contrôlabilité de l'équation d'Euler tridimensionnelle pour les fluides parfaits incompressibles, Séminaire sur les Équations aux Dérivées Partielles, 1997-1998, Exp No XV. École Polytechnique, Palaiseau (1998) 11. 
[16] O. Glass, Contrôlabilité exacte frontière de l'équation d'Euler des fluides parfaits incompressibles en dimension 3. C.R. Acad. Sci. Paris Sér. I Math. (1997) 987-992.

17] L. Hörmander, Linear partial differential operators. Springer-Verlag, Berlin (1963).

[18] T. Horsin, On the controllability of the Burgers equations. ESAIM: COCV 3 (1998) 83-95.

[19] O.Yu. Imanuvilov, On exact controllability for the Navier-Stokes equations. ESAIM: COCV 3 (1998) 97-131.

[20] O.Yu. Imanuvilov, Boundary controllability of parabolic equations. Sb. Math. 186 (1995) 879-900.

[21] O.Yu. Imanuvilov, Local exact controllability for the 2-D Navier-Stokes equations with the Navier slip boundary conditions. Lecture Notes in Phys. 491 (1977) 148-168.

[22] O.Yu. Imanuvilov and M. Yamamoto, On Carleman inequalities for parabolic equations in Sobolev spaces of negative order and exact controllability for semilinear parabolic equations, UTMS 98-46.

[23] A.N. Kolmogorov and S.V. Fomin, Introductory real analysis. Dover Publications, INC, New York (1996).

[24] O.A. Ladyzenskaja and N.N. Ural'ceva, Linear and quasilinear equations of elliptic type. Academic Press, New York (1968).

[25] J.L. Lions, Contrôle des systèmes distribués singuliers. Gauthier-Villars, Paris (1983).

[26] J.L. Lions, Optimal control of systems governed by partial differential equations. Springer-Verlag (1971).

[27] J.-L. Lions, Are there connections between turbulence and controllability?, in $9^{e}$ Conférence internationale de l'INRIA. Antibes (1990).

[28] J.-L. Lions and E. Magenes, Non-Homogeneous Boundary Value Problems. Springer-Verlag, Berlin (1971).

[29] M. Taylor, Pseudodifferential operators. Princeton Univ. Press (1981).

[30] M. Taylor, Pseudodifferential operators and Nonlinear PDE. Birkhäuser (1991).

[31] R. Temam, Navier-Stokes equations. North-Holland Publishing Company, Amsterdam (1979). 